OPEN ACCESS

Edited by:

Dirk Feldmeyer,

Helmholtz Association of German Research Centers (HZ), Germany

Reviewed by:

Michael Beierlein, University of Texas Health Science Center at Houston, United States Kimberly M. Huber, University of Texas Southwestern Medical Center, United States

*Correspondence: John M. Bekkers john.bekkers@anu.edu.au

Received: 21 December 2019 Accepted: 01 April 2020 Published: 30 April 2020

Citation:

Bekkers JM (2020) Autaptic Cultures: Methods and Applications. Front. Synaptic Neurosci. 12:18. doi: 10.3389/fnsyn.2020.00018

\section{Autaptic Cultures: Methods and Applications}

\begin{abstract}
John M. Bekkers*
Eccles Institute of Neuroscience, The John Curtin School of Medical Research, The Australian National University, Canberra, ACT, Australia

Neurons typically form daisy chains of synaptic connections with other neurons, but they can also form synapses with themselves. Although such self-synapses, or autapses, are comparatively rare in vivo, they are surprisingly common in dissociated neuronal cultures. At first glance, autapses in culture seem like a mere curiosity. However, by providing a simple model system in which a single recording electrode gives simultaneous access to the pre- and postsynaptic compartments, autaptic cultures have proven to be invaluable in facilitating important and elegant experiments in the area of synaptic neuroscience. Here, I provide detailed protocols for preparing and recording from autaptic cultures (also called micro-island or microdot cultures). Variations on the basic procedure are presented, as well as practical tips for optimizing the outcomes. I also illustrate the utility of autaptic cultures by reviewing the types of experiments that have used them over the past three decades. These examples serve to highlight the power and elegance of this simple model system, and will hopefully inspire new experiments for the interrogation of synaptic function.
\end{abstract}

Keywords: autapse, culture, methods, review, synapse

\section{INTRODUCTION}

The brain achieves its astonishing feats of information processing in part because of the complexity of its synaptic connections. Many synaptic circuit motifs have been elucidated, including feedforward, feedback, recurrent and lateral inhibition and excitation (Douglas and Martin, 2007; Yuste, 2015). Perhaps surprisingly, one of the most simple circuit motifs of all-that in which a neuron makes a synaptic connection with itself-was relatively late to come to the attention of neuroscientists. The term "autapse" entered the neuroscience lexicon only in 1972 when it was first coined to describe putative self-synapses on Golgi-stained pyramidal neurons in rabbit neocortex (Van der Loos and Glaser, 1972). Since then, both anatomical and physiological evidence for autapses in vivo has accumulated steadily (Karabelas and Purpura, 1980; Park et al., 1980; Peters and Proskauer, 1980; Preston et al., 1980; Lübke et al., 1996; Cobb et al., 1997; Tamás et al., 1997; Pouzat and Marty, 1998, 1999; Pawelzik et al., 2003; Bacci and Huguenard, 2006; Connelly and Lees, 2010; Manseau et al., 2010; Jiang et al., 2012, 2015; Yin et al., 2018; Deleuze et al., 2019). Today there is no question that autapses exist in the brain, albeit in much smaller numbers than (hetero-) synapses. However the importance of autapses for the normal operation of neural circuits remains a matter for speculation (Bekkers, 1998, 2003, 2009; White et al., 1998; Li et al., 2010; Connelly, 2014; Deleuze et al., 2014; Guo et al., 2016; Wiles et al., 2017). 
In parallel with these discoveries about autapses in intact brain tissue, it was found that, under the right conditions, autapses in neuronal cultures are surprisingly common. When constrained to grow in isolation on "microislands" or "microdots" a few tens of microns across (Furshpan et al., 1976, 1986; Landis, 1976), cultured neurons readily form autapses (Segal and Furshpan, 1990; Bekkers and Stevens, 1991). The presence of two or more neurons on the microisland does not seem to curtail autapse formation (Tarsa and Goda, 2002; Wierda and Sørensen, 2014), suggesting that neurons are just as likely to form autapses as synapses when given the opportunity. It is possible that the 2-dimensional geometry of cultures, with the greater likelihood that an axon will encounter its dendrites, is an important reason why autapses are so prevalent in culture (Ikeda and Bekkers, 2006).

Although initially a curiosity, autaptic cultures have proven to be a valuable model system for addressing a range of important questions in cellular neuroscience. By providing a homogeneous population of synaptic contacts on a single, isolated neuron, autaptic cultures offer the ultimate in synaptic reductionism. Their functional simplicity has enabled many important and elegant experiments that would not have been possible in more complex systems.

The goals of this article are, first, to show how to prepare and utilize these cultures and, second, to give an overview of their many applications, with an emphasis on neurophysiological experiments. An alternative method for studying single neurons in isolation is to use very-low-density cultures, which have been described in detail elsewhere (e.g., Goslin et al., 1998; Ventimiglia and Lindsay, 1998; Fath et al., 2009); I will not be discussing this approach here. I will also not be discussing the use of similar cultures to study neurite growth on patterned substrates, which is of interest to the design of brain-machine interfaces (e.g., Jang et al., 2016; Gautam et al., 2017).

\section{METHODS FOR PREPARING AUTAPTIC CULTURES}

Autaptic cultures are prepared in essentially the same way as conventional dissociated primary cultures ("mass cultures"), the main difference being the preparation of the culture plates. Several excellent articles about preparing autaptic cultures have been published (Segal et al., 1998; Allen, 2006; Fasano et al., 2008; Rost et al., 2010; Burgalossi et al., 2012; Lu et al., 2016) and the guidance below draws upon all of these, as well as my own experience (Bekkers and Stevens, 1991; Bekkers, 2005). It should be kept in mind that cell culture is often laced with superstition. The best advice is to start simple and elaborate only if necessary.

The methods presented here will focus on general-purpose hippocampal or cortical cultures prepared from newborn mice or rats. Others have described how to prepare cultures from embryos (Fath et al., 2009; Lu et al., 2016), older tissue (Ogata and Tatebayashi, 1991; Brewer, 1997; Allen, 2006) and other brain areas (Johnson, 1994; Shi and Rayport, 1994; Sulzer et al., 1998; Michel and Trudeau, 2000; Moechars et al., 2006). There is even a protocol for preparing autaptic cultures from human induced pluripotent stem cells (Fenske et al., 2019). Whatever the tissue source, it goes without saying that all procedures must be approved by the local ethics committee.

\section{Overview of the Procedure}

Figure 1 shows the basic steps in preparing autaptic cultures, and the detailed requirements are listed in Tables 1-4. Briefly, the key initial step is to prepare coverslips with spots of permissive growth substrate (e.g., collagen, poly-D-lysine) dispersed across a coating of non-permissive substrate (agarose). Dissociated primary neurons are then added to the coverslips, usually (but not always) after first growing a monolayer of glial cells (astrocytes) on the spots to provide trophic support for the neurons. Autaptic neurons are typically ready for use after 1-2 weeks in vitro. The entire procedure will take at least 2-3 weeks, depending on the exact method used.

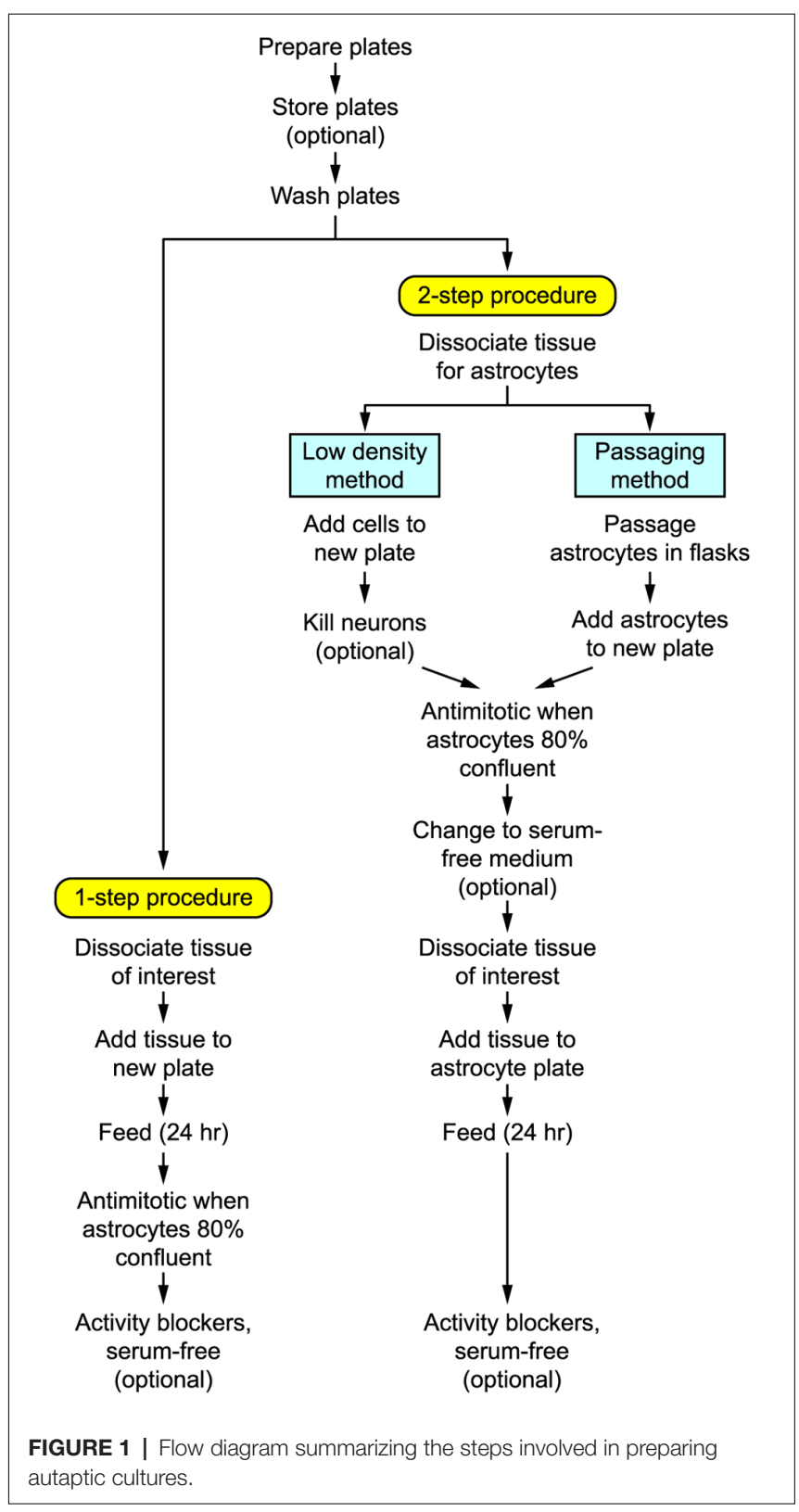


TABLE 1 | Solutions for preparing plates.

\section{Permissive coating solution}

Stock solutions:

- $5 \mathrm{mg} / \mathrm{ml}$ poly-D-lysine in sterile $\mathrm{dH}_{2} \mathrm{O}$

- Collagen as assayed by the supplier

To prepare $1 \mathrm{ml}$ of permissive coating solution:

- Aim for final concentrations of $\sim 0.1 \mathrm{mg} / \mathrm{ml}$ poly-D-lysine and $\sim 0.5 \mathrm{mg} / \mathrm{ml}$ collagen

- Dilute the collagen to $0.5 \mathrm{mg} / \mathrm{ml}$ in $1 \mathrm{ml}$ of sterile $\mathrm{dH}_{2} \mathrm{O}$

- Add $20 \mu \mathrm{l}$ of poly-D-lysine stock to the $1 \mathrm{ml}$ of diluted collagen

Keep the coating solution for several weeks at $4^{\circ} \mathrm{C}$.

\begin{tabular}{lll}
\hline Quantity & Name & Supplier \\
\hline $5 \mathrm{mg}$ & Poly-D-lysine hydrobromide 30-70K & Sigma-Aldrich \\
$20 \mathrm{ml}$ & Rat tail collagen & Thermo Fisher \\
$5 \mathrm{~g}$ & Agarose, Type II-A: medium EEO & Sigma-Aldrich \\
& Sigma-Aldrich
\end{tabular}

Notes: 1. Poly-L-lysine, and either the hydrobromide or hydrochloride salt, should work as well, but some experimentation may be required. 2. Some investigators recommend preparing the poly-D/L-lysine stock in borate buffer (1.24 g of boric acid, $1.9 \mathrm{~g}$ of sodium tetraborate, $400 \mathrm{ml}$ of $\mathrm{H}_{2} \mathrm{O}$ ).

TABLE 2 | Solutions for tissue dissociation.

\section{Dissection solution}

This can be standard mammalian Ringer containing (in mM): $125 \mathrm{NaCl}, 3 \mathrm{KCl}, 2 \mathrm{CaCl}_{2}, 1 \mathrm{MgCl} 2,25 \mathrm{HEPES} @ \mathrm{pH} 7.4$ adjusted with NaOH, 10 glucose, 25 sorbitol to give $315 \mathrm{mOsm} / \mathrm{kg}, 0.22 \mu \mathrm{m}$ filtered to sterilize.

\section{Enzyme solution}

Mix in a $15 \mathrm{ml}$ tube:

- 5 ml Hank's Balanced Salt Solution (BSS; divalent-free, with phenol red)

- 50 ul 50 mM EDTA stock

- $75 \mu \mathrm{l} 100 \mathrm{mM} \mathrm{CaCl}_{2}$

- $\sim 1 \mathrm{mg}$ cysteine

- 100 units papain suspension

Add about $10 \mu$ l of $1 \mathrm{M} \mathrm{NaOH}$ to adjust $\mathrm{pH}$ to about 7 (i.e., a pale pink color).

Keep in $37^{\circ} \mathrm{C}$ bath for $10-15 \mathrm{~min}$ to dissolve papain, then $0.22 \mu \mathrm{m}$ filter into another sterile $15 \mathrm{ml}$ tube and return the tube to the $37^{\circ} \mathrm{C}$ bath until required.

\begin{tabular}{|c|c|c|c|}
\hline Quantity & Name & Supplier & Catalog number \\
\hline $500 \mathrm{ml}$ & Hank's BSS, divalent-free, with phenol red & Thermo Fisher & 14170112 \\
\hline $100 \mathrm{~g}$ & EDTA & Sigma-Aldrich & E6758 \\
\hline $5 \mathrm{~g}$ & L-cysteine, $\mathrm{HCl}$ monohydrate & Sigma-Aldrich & C7889 \\
\hline \multirow[t]{2}{*}{$100 \mathrm{mg}$} & Papain, suspension & Worthington & LS 003126 \\
\hline & & Sigma-Aldrich & P3125 \\
\hline
\end{tabular}

\section{Preparation of Plates}

(1) Clean the coverslips. We use 24-well culture plates and grow the cells on $12-13 \mathrm{~mm}$ diameter No. 1 circular glass coverslips, one per well, which routinely provides 1-5 isolated single neurons per coverslip, i.e., up to this number of autapse experiments per well. Larger coverslips may be more convenient in some situations (see Step 5). Clean the coverslips by swirling them for $5 \mathrm{~min}$ in $10 \mathrm{~N}$ nitric or hydrochloric acid. This is intended to make the glass more hydrophilic for the agarose coating, but simply cleaning with ethanol also works (Lu et al., 2016). Rinse thoroughly in tap water, followed by distilled water then $100 \%$ ethanol, and store in a glass bottle under $100 \%$ ethanol. A large number can be cleaned at the same time and used for months.

(2) Place coverslips in culture plates. Using forceps and working in a sterile hood, flame the coverslips dry one at a time by passing them through a gas flame. Place the coverslips in the culture plates, one per well. Center the coverslip in each well and press down gently to "stick" it in position.
(3) Coat coverslips with agarose. Prepare $0.15-0.2 \%$ agarose in $\mathrm{dH}_{2} \mathrm{O}$. This can be done by weighing 15-20 $\mathrm{mg}$ of agarose into $10 \mathrm{ml}$ of $\mathrm{dH}_{2} \mathrm{O}$ in a $15 \mathrm{ml}$ centrifuge tube. The tube, with its cap loosened, is microwaved or placed in boiling water for 2-5 min until the agarose is dissolved. Using a $200 \mu \mathrm{l}$ pipette, spread a thin layer of the agarose solution on each coverslip then suck off the excess (It helps to first stick down the coverslip by placing a drop of solution at the edge of the coverslip so it runs underneath). It is important that the whole coverslip is covered with an agarose solution; any gaps can allow astrocytes or neurons to grow on the glass, compromising the required isolation of microislands. Sometimes the glass does not wet well; in this case, you need to add more agarose solution so a large drop completely covers the glass. Allow the plate to dry in the hood. We usually prepare 4 or 5 plates like this at once then store them at room temperature for several months (Burgalossi et al., 2012). 
TABLE 3 | Culture media.

\section{Classic serum-containing medium}

To prepare $100 \mathrm{ml}$ :

- $2 \mathrm{ml}$ of $1 \mathrm{M}$ stock glucose in MEM with Earle's Salts

- $5 \mathrm{ml}$ of heat-inactivated Fetal Bovine Serum (FBS)

- $1 \mathrm{ml}$ of 5,000 units/ml stock Penicillin-Streptomycin

- MEM, Earle's salts, top-up to $100 \mathrm{ml}$

- $100 \mu \mathrm{l}$ Serum Extender

Filter $(0.22 \mu \mathrm{m})$ into a sterile bottle. Keep in the dark at $4^{\circ} \mathrm{C}$ for about 2 weeks.

\begin{tabular}{|c|c|c|c|}
\hline Quantity & Name & Supplier & Catalog number \\
\hline $1,000 \mathrm{ml}$ & Minimal Essential Medium (MEM) with Earle's Salts, without glutamine & Sigma-Aldrich & $51412 \mathrm{C}$ \\
\hline $100 \mathrm{ml}$ & FBS & Various & \\
\hline $100 \mathrm{ml}$ & Penicillin-Streptomycin & Thermo Fisher & 15070063 \\
\hline 1 vial & MITO+ Serum Extender & Corning & 355006 \\
\hline
\end{tabular}

Alternative serum-containing medium

To prepare $100 \mathrm{ml}$ :

- 50 ml DMEM with HEPES: Weigh out 870 mg Dulbecco's Modified Eagle's Medium (DMEM) with high glucose (powder), add <50 ml distilled water (dH ${ }_{2} \mathrm{O}$ ) while stirring. Make up to $50 \mathrm{ml}$ with $\mathrm{dH}_{2} \mathrm{O}$.

- Mix the $50 \mathrm{ml}$ prepared in step 1 with $50 \mathrm{ml}$ of DMEM with high glucose (liquid).

- 2 ml B-27 supplement.

- $1 \mathrm{ml}$ of 5,000 units/ml stock Penicillin-Streptomycin.

- $5 \mathrm{ml}$ heat-inactivated FBS.

- Add about $350 \mu \mathrm{l} 1 \mathrm{M} \mathrm{NaOH}$ to adjust pH to about 7.5.

Filter $(0.22 \mu \mathrm{m})$ into a sterile bottle. Keep in the dark at $4^{\circ} \mathrm{C}$ for about 2 weeks.

\begin{tabular}{|c|c|c|c|}
\hline Quantity & Name & Supplier & Catalog number \\
\hline For $10 \mathrm{~L}$ & Dulbecco's Modified Eagle's Medium with high glucose (powder) & Sigma-Aldrich & D1152 \\
\hline $100 \mathrm{ml}$ & Dulbecco's Modified Eagle's Medium with high glucose (liquid) & Sigma-Aldrich & D0422 \\
\hline $10 \mathrm{ml}$ & B-27 Supplement $(50 \times)$ & Thermo Fisher & 17504044 \\
\hline $100 \mathrm{ml}$ & Penicillin-Streptomycin & Thermo Fisher & 15070063 \\
\hline $100 \mathrm{ml}$ & FBS & Various & \\
\hline
\end{tabular}

Complete Neurobasal medium (serum-free)

To prepare $100 \mathrm{ml}$, mix aseptically:

- $98 \mathrm{ml}$ of Neurobasal or Neurobasal Plus medium

- 2 ml of B-27 or B-27 Plus Supplement

- $250 \mu$ l of GlutaMAX-I Supplement

Keep in the dark at $4^{\circ} \mathrm{C}$ for about 2 weeks.

\begin{tabular}{|c|c|c|c|}
\hline Quantity & Name & Supplier & Catalog number \\
\hline \multirow[t]{2}{*}{$500 \mathrm{ml}$} & Neurobasal Medium & Thermo Fisher & 21103049 \\
\hline & Neurobasal Plus medium & & A3582901 \\
\hline \multirow[t]{2}{*}{$10 \mathrm{ml}$} & B-27 Supplement $(50 \times)$ & Thermo Fisher & 17504044 \\
\hline & B-27 Plus Supplement (50x) & & A3582801 \\
\hline $100 \mathrm{ml}$ & GlutaMAX-I Supplement & Thermo Fisher & A1286001 \\
\hline
\end{tabular}

Notes: 1. To heat-inactivate FBS, heat @ $56^{\circ} \mathrm{C}$ for $30 \mathrm{~min}$ in a water bath. 2. MEM without phenol red may be preferred for cultures that will be used for imaging experiments to minimize background fluorescence. 3. For suppressing the overgrowth of glial cells, prepare a $2.5 \mathrm{mM}$ stock solution of cytosine arabinoside, free base (Sigma-Aldrich, C-1768) in $\mathrm{dH}_{2} \mathrm{O}$ and store the stock at $-20^{\circ} \mathrm{C}$. Dilute this in warmed culture medium to achieve a final concentration of $5 \mu \mathrm{M}$ in the culture plate after feeding. 4 . Glial cells grow more readily in medium supplemented with FBS, so an alternative for suppressing glial growth, particularly if plating onto an astrocyte feeder layer, is to replace the FBS with twice the concentration of horse serum.

(4) Prepare the permissive substrate. Prepare about $1 \mathrm{ml}$ of permissive substrate solution. We normally use a mixture of poly-D-lysine and rat tail collagen (Table 1) because cells attach better to poly-D-lysine (Segal et al., 1998) but collagen adds viscosity that may help with the spotting (next step); however, either could be used on its own. The mixture keeps for several weeks at $4^{\circ} \mathrm{C}$. Possible alternatives are poly-L-lysine and poly-D,L-ornithine (Segal et al., 1998).

(5) Apply spots of permissive substrate. The next step-dispersing small spots of poly-D-lysine/collagen on the agarose coating-is critical. The original method uses a micro atomizer or airbrush to spray a mist of permissive substrate onto the agarose-coated coverslips, which requires judgment and experience to achieve a satisfactory distribution of small, discrete spots (Segal and Furshpan, 1990; Bekkers and Stevens, 1991; Fasano et al., 2008). A more recent method is to fabricate a stamping tool that imprints a regular pattern of permissive substrate solution onto the coverslips (Moulder et al., 2007; Sgro et al., 2011; Burgalossi et al., 2012; Ricoult et al., 2012).

(i) Microatomizer method. Obtain a small glass reagent sprayer or artist's airbrush (see Table 4 for suggested 
TABLE 4 | Other requirements.

$13 \mathrm{~mm}$ round glass coverslips, No. 1 (0.13-0.17 mm thickness), cleaned as described

24 well culture plates

$56 \mathrm{~mm}$ sterile culture dish (for coarse dissection of tissue)

$35 \mathrm{~mm}$ sterile culture dish (for fine dissection of tissue, if required)

$15 \mathrm{ml}$ sterile plastic centrifuge tubes (for preparing solutions, incubating in enzyme, trituration)

$50 \mathrm{ml}$ sterile plastic centrifuge tubes (for working aliquots of culture medium)

$0.22 \mu \mathrm{m}$ pore $25 \mathrm{~mm}$ diameter sterile syringe filters (for sterilizing solutions)

$10 \mathrm{ml}$ syringes (for use with syringe filters)

$10 \mathrm{ml}$ sterile pipettes (for dispensing medium and adding cell suspension to plates)

Sterile Pasteur pipettes plugged at the wide end with cotton wool (for dispensing solutions during dissection and doing trituration)

Hemocytometer and access to a microscope (for counting cells)

Dissection instruments: e.g., dissection scope, medium scissors for decapitation, small scissors for opening the skull, small flat spatula for removing the brain and doing coarse dissection, one pair fine forceps, one scalpel blade for mincing tissue

$80 \% \mathrm{v} / \mathrm{v}$ ethanol in distilled water (for sterilizing the dissection instruments and work area)

Sterile bottles (for storing the culture medium and solutions used in the preparation)

Small atomizer (for spraying the culture plates). We have the most experience with a small glass reagent sprayer (e.g., Kimble 5 ml thin layer chromatography sprayer, kimble-chase.com) but a small recycled perfume spray bottle might work as well, or an artist's airbrush (e.g., Aztec airbrush from www.testors.com). See "Preparation of Plates" section for more details.

Stamping tool for applying spots of permissive substrate. This is an alternative to the atomizer. The stamping tool yields more reproducible microislands but the tool needs to be fabricated. See "Preparation of Plates" section for more details.

Notes: 1. Quantities depend on the number of cultures. We find that one newborn animal is sufficient to yield enough hippocampal neurons for one or two 24-well plates. 2. The composition of the glass used in the coverslips may affect the health of the cultures. Be prepared to test coverslips from different suppliers.

sources). A small perfume bottle may also work. Sterilize the sprayer by spraying $80 \%$ ethanol and allowing it to dry in the culture hood. Load the substrate solution into the atomizer and spray the coating solution onto the dry agarose-coated plates. The spraying pressure, spraying duration, and distance from the plate need to be optimized so that small, discrete spots $(\sim 200 \mu \mathrm{m}$ diameter) of the substrate solution settle on the coverslip. Here are some tips to guide mastery of this critical step (see also "Troubleshooting” section below).

- Hold the culture plate vertically (the coverslips should not fall out if they have been stuck down with agarose) against a dark surface for better visibility then apply a brief burst of spray from about $15 \mathrm{~cm}$ away to cover each half of the plate.

- Alternatively, place the plates on a horizontal surface and spray from $\sim 50 \mathrm{~cm}$ away, allowing the mist to settle on the plates (Fasano et al., 2008).

- Higher pressure in the atomizer tends to produce smaller spots, but the spraying time needs to be brief to avoid producing too many spots that merge.

- The spattering can be practiced by spraying an uncoated coverslip with a test solution in which a dye (e.g., Trypan blue) is added to the normal coating solution (to have the right viscosity). The distribution and sizes of the spots can be observed under a dissecting scope immediately after spraying, then the spots can then be wiped off and another attempt made.

- The spots may vary markedly in size, which is acceptable; you only need $\sim 10-20$ spots on the coverslip that have the preferred size $(\sim 200 \mu \mathrm{m})$. (ii) Stamping tool method. This method requires the fabrication of a special "microstamp" but yields much more reproducible microislands and, hence, a higher yield of isolated autaptic neurons (Moulder et al., 2007; Rost et al., 2010; Sgro et al., 2011; Burgalossi et al., 2012; Ricoult et al., 2012). The stamp can be made using photolithography and microcontact printing techniques. A typical pattern might be an array of squares or circles, $150-200 \mu \mathrm{m}$ across, spaced at 400-500 $\mu \mathrm{m}$ intervals (Sgro et al., 2011; Burgalossi et al., 2012). The permissive substrate solution is loaded onto the stamp then transferred to the agarose-coated coverslips with gentle pressure. The key precaution with this method is to avoid under- or overloading the stamp with substrate solution (Moulder et al., 2007; Burgalossi et al., 2012).

(6) Sterilize and store the prepared plates. Allow the plates to dry in the hood, which should only take a few minutes. It is advisable to sterilize the plates by placing them under a UV lamp in the culture hood for 20-30 min. Prepared plates can then be covered and kept at room temperature for several weeks before use. Indeed, doing so has been reported to result in healthier cultures (Sgro et al., 2011), although others state that the plates should be prepared just before use (Fath et al., 2009).

(7) Wash the plates. The day before the cell preparation, add a few drops of culture medium to each well-just enough to cover the coverslip-and keep the plates in an incubator overnight. This "washes" the substrate and seems to improve the survival of cells. Just before adding the cell suspension to the plates the washing medium is sucked out and discarded. 
A variation on the above is to use the "sandwich" method, in which the coverslip containing the neurons is opposed to and separate from an astrocyte feeder culture that provides diffusible growth factors to the medium (Brewer and Cotman, 1989). A simple way to prepare plates for this method is to simply scratch the bottom of each well with a needle ( $\mathrm{Lu}$ et al., 2016). This raises small protuberances of plastic that will hold the coverslip just above the bottom of the well. Another method is to briefly touch the bottom of each well in three places with a hot soldering iron, raising small plastic welts. The coverslips for "sandwich" cultures are prepared by first distributing them in large (e.g., $60 \mathrm{~mm}$ ) culture dishes. They are then coated with agarose and sprayed with a permissive substrate, as described above for the standard method. Finally, they are sterilized and stored in the large culture dishes until needed for the cell preparation. See "Alternative Plating Method" section below for further details.

\section{Plating Procedure}

Broadly speaking, primary neuronal cultures can be prepared in two different ways (Figure 1):

(i) One-step procedure. Neural tissue is enzymatically dissociated and the single-cell suspension is plated at a high enough density that the neurons will survive while the astrocytes become sufficiently numerous to provide longer-term trophic support for the neurons. This method works best with rats or embryonic tissue.

(ii) Two-step procedure. A monolayer "lawn" of astrocytes without neurons is prepared first, then the neurons are plated on top of the astrocytes 1-3 weeks later. This method works well for both rat and mouse postnatal tissue and tends to give a more consistent yield of autaptic neurons (Pyott and Rosenmund, 2002). The astrocytic lawn can be prepared in two ways.

(a) Low-density: The dissociated cell suspension is directly added to the microisland plates at a low enough density that only the astrocytes survive.

(b) Passaging: The astrocytes are grown and passaged separately before adding them to the microisland plates.

For both the one-step and two-step procedures the cultures are more likely to be successful when prepared from embryonic or newborn tissue, e.g., from rodents aged between embryonic day 16 (E16) and postnatal days $0-3(\mathrm{P} 0-3)$. If cultures need to be prepared from older animals, more elaborate procedures are required (Kay and Wong, 1986; Kaneda et al., 1988; Ogata and Tatebayashi, 1991; Brown et al., 1993; Magistretti et al., 1996; Brewer, 1997; Allen, 2006). The steps presented below assume that newborn rodent tissue is being used. Methods for using embryonic tissue are described elsewhere (Goslin et al., 1998; Fath et al., 2009).

Unless otherwise stated, all of the following steps are done in a sterile laminar flow hood.

(1) Prepare instruments and solutions. Assemble the dissection instruments (Table 4) in the hood and sterilize them with $80 \%$ ethanol. Prepare the enzyme solution (Table 2), chill the dissection solution (Table 2) and warm the culture medium (Table 3). Papain is most commonly used in the enzyme solution because it is regarded as gentler than trypsin for dissociating fragile neural tissue (Goslin et al., 1998). However, if preparing astrocytes for passaging (see Step 8 below) then trypsin is suitable and more economical. The choice of the culture medium is critical. For growing neurons under defined conditions, serum-free medium (e.g., Neurobasal medium; Table 3) is preferred. On the other hand, astrocytes grow better in serum-containing medium (Table 3, Rost et al., 2010; Burgalossi et al., 2012). Thus, unless the neurons are being plated onto a confluent monolayer of astrocytes (see Steps 6 and 8 below), it is advisable to start with a serum-containing medium to promote astrocyte division. If desired, the culture can be switched to a serum-free medium after the astrocytes have reached confluence. A disadvantage of using serum is that there may be batch-to-batch variability in the properties of the serum, and so it may be advisable to test small amounts of different batches before placing a larger order.

(2) Extract the tissue. One newborn rat or mouse pup provides enough hippocampal or cortical cells for one or two 24-well plates. Sacrifice the pup using an approved method of euthanasia and remove the brain into ice-cold dissection solution. Dissect out the hippocampi or cortical tissue, if possible peel off the meninges (enclosing membrane), then cut the tissue into roughly $1 \mathrm{~mm}$ square blocks.

(3) Incubate the tissue in enzyme. Using a sterile pipette, transfer the tissue pieces, with as little of the dissection solution as possible, to the sterile $15 \mathrm{ml}$ centrifuge tube containing the enzyme solution. Place the tube in a $37^{\circ} \mathrm{C}$ water bath with agitator and leave it gently agitating for $30 \mathrm{~min}$. Alternatively, gentle manual agitation for a few seconds every 10 min will suffice. While the tissue is incubating, prepare a plugged sterile glass Pasteur pipette by gently melting the tip of the pipette in a burner flame in the hood. You want to smoothen the sharp edges of the pipette tip without making the tip much narrower.

(4) Dissociate the tissue. At the end of the incubation, move the tube with tissue back to the laminar flow hood and (optionally) add $50-100 \mu \mathrm{l}$ of $10 \mathrm{mg} / \mathrm{ml}$ DNAse I stock solution and wait for about $30 \mathrm{~s}$. This breaks down DNA from damaged cells and reduces clumping during the subsequent trituration. Using the smoothed Pasteur pipette, suck off as much as possible of the supernatant enzyme solution and add 1-2 $\mathrm{ml}$ of warmed culture medium. After allowing the tissue to settle, suck off and discard the supernatant. Repeat this 3-4 times to completely wash out the enzyme. Add a milliliter or so of culture medium and very gently triturate the tissue 3-4 times. Wait for the pieces to settle, then save the supernatant (which should appear a little cloudy due to the suspension of dissociated cells) to another sterile $15 \mathrm{ml}$ tube. Repeat this process 6-8 times, each time triturating slightly more vigorously (but still very gently) and saving the supernatant until the tissue is mostly dissociated. Ensure that no air bubbles get into the solution at any 
stage during this procedure. Being very gentle and careful is the key to success. You should finish with 6-10 $\mathrm{ml}$ of single-cell suspension.

(5) Count the cells. Counting is done using a hemocytometer on a phase-contrast microscope. A good preparation is one that contains many phase-bright neurons with the stumps of processes visible. However, we count all phase-bright cells, including ones without obvious processes. Typically we get a yield of about $15-25 \times 10^{4}$ cells $\mathrm{ml}^{-1}$ from the CA1 regions of two hippocampi. Possible remedies for a low yield are given in the "Troubleshooting" section.

(6) Determine the dilutions. Table 5 gives suggested cell counts and volumes for different types of cultures. When using the two-step procedure we typically prepare two 24-well plates during each culture preparation: one in which we prepare a low-density astrocyte culture using a new culture plate ( $C$ or $D$ in Table 5), and one in which we plate neurons on top of a low-density astrocyte culture that was prepared 2-3 weeks previously and which is now a confluent monolayer ( $E$ or $F$ in Table 5). Note that, when plating on top of an astrocyte monolayer ( $E$ or $F$ ), the suspension of neurons is simply added to the $0.5 \mathrm{ml}$ of the medium that is already in each well. The low-density astrocyte cultures ( $C$ and $D$ in Table 5) typically grow to confluency over 2-3 weeks with little contamination from surviving neurons. However, if neurons do remain, they can usually be lysed by simply removing the plate from the incubator for 30-60 min. Alternatively, the neurons can be killed by adding $0.2-1 \mathrm{mM}$ glutamate to the culture medium for several hours, then rinsing and refeeding with fresh culture medium (Segal et al., 1998; Harms et al., 2005). Note that cell plating densities are advisory, and experimentation will be required to find densities that suit your particular conditions. In general, higher plating density leads to more surviving neurons with greater synaptic connectivity (Ivenshitz and Segal, 2010), but this may be a disadvantage if the aim is to maximize the number of microdots occupied by a single neuron. Further comments on plating density are made in the sections "Troubleshooting" and "Electrophysiological Recordings From Autapses."

(7) Incubate the culture plates. Place the culture plate(s) into an incubator at $37^{\circ} \mathrm{C}, 5 \% \mathrm{CO}_{2}$. The neurons should settle and

\section{TABLE 5 | Cell dilutions.}

\begin{tabular}{|c|c|c|}
\hline Type of culture & $\begin{array}{l}\text { Final cell density } \\
\qquad\left(\times 10^{4} \mathrm{ml}^{-1}\right)\end{array}$ & $\begin{array}{l}\text { Volume per well } \\
\text { (24-well plate; } \mathrm{ml})\end{array}$ \\
\hline A: 1-step procedure, rat & $7-9$ & 0.5 \\
\hline$B:$ 1-step procedure, mouse & $12-15$ & 0.5 \\
\hline $\begin{array}{l}\text { C: 2-step procedure, rat, to prepare } \\
\text { a low-density astrocyte culture }\end{array}$ & $3-5$ & 0.5 \\
\hline $\begin{array}{l}D: \text { 2-step procedure, mouse, to } \\
\text { prepare a low-density astrocyte } \\
\text { culture }\end{array}$ & $3-5$ & 0.5 \\
\hline $\begin{array}{l}\text { E: 2-step procedure, rat, plating on } \\
\text { an astrocyte monolayer }\end{array}$ & $6-10$ & 0.1 \\
\hline F: 2-step procedure, mouse, plating & $10-15$ & 0.1 \\
\hline
\end{tabular}

stick to the substrate within an hour or two. They should begin to extend processes within $24 \mathrm{~h}$, or sooner if plated on astrocytes.

(8) Passaging method for preparing astrocyte monolayers. Passaging provides an ample supply of pure astrocytes that can be plated out on new culture dishes at a higher density than in Step 6 above, which means that a confluent astrocytic "lawn" will grow more quickly. On the other hand, this approach is more complex. Briefly, neural tissue is dissociated as in Steps 1-4 above, except that harsher enzymes (e.g., trypsin) and more vigorous trituration are used to favor the more hardy astrocytes over neurons. The cells are grown in culture flasks under conditions that suit astrocytes and are passaged until required (Ullian et al., 2001).

\section{Feeding}

Following the plating of neurons, it is normal for significant neuronal death and accumulation of debris to occur over the first $24 \mathrm{~h}$. The debris should be removed as much as possible by feeding the culture the day after the dissociation. If using 24 -well plates, this is done by removing $0.15 \mathrm{ml}$ of the old medium from each well (i.e., about $1 / 3$ the well volume) and adding the same quantity of fresh, warmed medium.

A general rule of thumb is that autaptic cultures do better with minimal feeding, say, once every 1-2 weeks using the $0.15 \mathrm{ml}$ off/on protocol above. However, there are several important exceptions to this rule.

(i) When astrocytes approach confluence (one-step procedure). About 4-5 days after plating with the one-step procedure the astrocytes should be about $80 \%$ confluent. To prevent overgrowth of the neurons by astrocytes, the cultures should be treated with an antimitotic drug, e.g., cytosine arabinoside (araC, $5 \mu \mathrm{M}$ final concentration; see Table 3, Notes), administered using the one-third off/on method above. The cultures are then fed again 1-3 days later with drug-free medium. Both of these feeds, as well as subsequent ones, can be done using a serum-free medium if desired, provided the transition is done slowly to avoid shock to the neurons.

(ii) When astrocytes approach confluence (two-step procedure, preparing an astrocyte culture). Again, treatment with antimitotic should be done when the astrocytes reach about $80 \%$ confluence. In this case, the switch to a serum-free medium could be made by exchanging all of the medium at once, taking advantage of the greater robustness of astrocytes. However, it is important not to change the medium too soon (e.g., $<2$ days) before neurons are plated onto the astrocytes; the astrocytes need to be given time to "condition" the medium with trophic factors that aid neuron survival. The antimitotic should be left in place until after the neuronal suspension is added, to prevent mitosis of the newly added astrocytes.

(iii) When the culture medium becomes acidic. If the medium contains phenol red, acidity is apparent as a shift to a pale 
yellow color, indicative of an accumulation of acidic waste products. This change is often seen in older, denser cultures. If this occurs, feeding should be done more frequently to minimize damage to the neurons.

Particularly in older cultures, it may help to add blockers of synaptic transmission to the culture medium to reduce excitotoxicity. For example, after 7-10 days in vitro we sometimes add kynurenic acid ( $1 \mathrm{mM}$ final concentration in each well), CNQX or DNQX $(10 \mu \mathrm{M})$ to inhibit non-NMDA ionotropic glutamate receptors, or D, L-APV $(40 \mu \mathrm{M})$ to inhibit NMDA receptors. We find that cultures typically survive much better for longer periods (>3-4 weeks) in the presence of one of these blockers. However, it should be kept in mind that chronic blockade of excitatory synaptic transmission may affect synapse maturation (Murthy et al., 2001).

\section{Alternative Plating Method}

The "sandwich" culture is an elegant method for exposing neurons to astrocyte-conditioned medium without physical contact with the astrocytes (Brewer and Cotman, 1989; Goslin et al., 1998; Lu et al., 2016). Because the autaptic neurons are resting on coated glass rather than an astrocytic lawn, the quality of the optics is improved, which is beneficial for certain experiments. In this method, dissociated cells are plated in wells that do not contain coverslips but which have small plastic protuberances on the bottom, as described in the "Preparation of Plates" section. After 1-2 weeks a confluent mixed neuron-glia feeder culture is obtained. At this time another dissociation is done and the isolated cells are plated onto coverslips that had previously been agarose-coated and sprayed in large culture dishes (see "Preparation of Plates" section and Goslin et al., 1998). These large culture dishes are placed in the incubator for a few hours to allow the neurons to settle and attach, then each coverslip is taken out, inverted, and placed neuron side down in each well in the plate containing the feeder culture. The plastic protuberances hold the coverslip and adherent neurons just above the glia, allowing access to secreted growth factors without direct contact.

\section{Troubleshooting}

(1) Agarose does not stick to the glass coverslips. Make sure the coverslips have been thoroughly washed in concentrated acid and just as thoroughly rinsed in water. We have found that it helps to store the coverslips under $100 \%$ ethanol. If the ethanol contains too much water, the glass seems to be less easily wetted by agarose.

(2) Problems with obtaining a suitable archipelago of microislands. When using the spray technique, it is unnecessary to be too fussy about getting uniform spot sizes. It is normal to have a wide variety of sizes and shapes, provided that the spots are at least a few tens of microns apart and there is a reasonable number of spots ( 10-20 per coverslip) that are roughly $200 \mu \mathrm{m}$ in diameter. The challenge is to find the optimum parameter settings for the sprayer being used. Parameters to vary include airflow, spray burst duration, distance of sprayer from the coverslips, and size of the spray nozzle. For example, with our glass microatomizer (Kimble-Chase; see Table 4) we use airflow (from a reticulated air supply) at

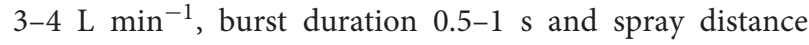
$\sim 10-15 \mathrm{~cm}$.

(3) Poor yield of cells following tissue dissociation. The dissociation is the critical step for obtaining good autaptic cultures and, indeed, any dissociated cultures. Three factors seem key to achieving a good yield of healthy neurons, these being in the order of decreasing importance: (i) the age of the animal; (ii) the care in trituration; and (iii) the choice of dissociation enzyme. In general, the younger the animal the better the yield of neurons and, hence, the better the quality of the culture. Even a single day can make a difference: in our experience, P0 pups provide better neurons than P1 or P2 animals. For the best neuronal yield, embryos (e.g., E16-18) can be used, but this comes at the cost of more challenging surgery and the loss of the mother (Fath et al., 2009). Trituration is another important factor and requires you to be slow, gentle and patient. Each stroke of the trituration should, for example, take several seconds. The supernatant containing the cell suspension should be collected after every 3-4 strokes to avoid exposing isolated cells to unnecessary additional trauma. The tip of the Pasteur pipette used for trituration should be carefully fire-polished in the flame of a gas burner. Some researchers recommend using two Pasteur pipettes with wider and narrower polished openings; the narrower tip is used later in the trituration process (Fath et al., 2009). Finally, the type and activity of the enzyme are important. We find that papain is best for P0-1 rat tissue, but the suitability of alternative enzymes for other tissues (e.g., trypsin; Allen, 2006; Fath et al., 2009) needs to be determined empirically. Be aware also that the assayed activity of an enzyme can decline with time in storage.

(4) Excessive neuronal death a few days after plating. As noted in the "Feeding" section some loss of neurons is normal within a day or two of plating, particularly if not plating on astrocytes. It is important to remove the debris by feeding the day after plating because the debris seems to have an inhibitory effect on the survival of the remaining cells. Subsequent excessive neuronal death could be due to a multitude of factors that can be time-consuming to track down. For convenience, it is best to troubleshoot these factors using mass cultures rather than microdots. Three key factors to explore are: (i) the substrate; (ii) the astrocyte feeder layer; and (iii) the culture medium (Goslin et al., 1998). Substrate problems might include the properties or cleanliness of the glass coverslips, or the freshness of the permissive coating solution. These can be tested by plating directly on the coated or uncoated bottom of plastic culture dishes, which are generally optimized for good cell survival. If the neurons survive better on plastic, test the coating solution on different coverslips. Anecdotally, problems have been traced back to the type of glass used in different brands of coverslips, although this is less likely to be an issue for autaptic cultures where the glass is covered with agarose. 

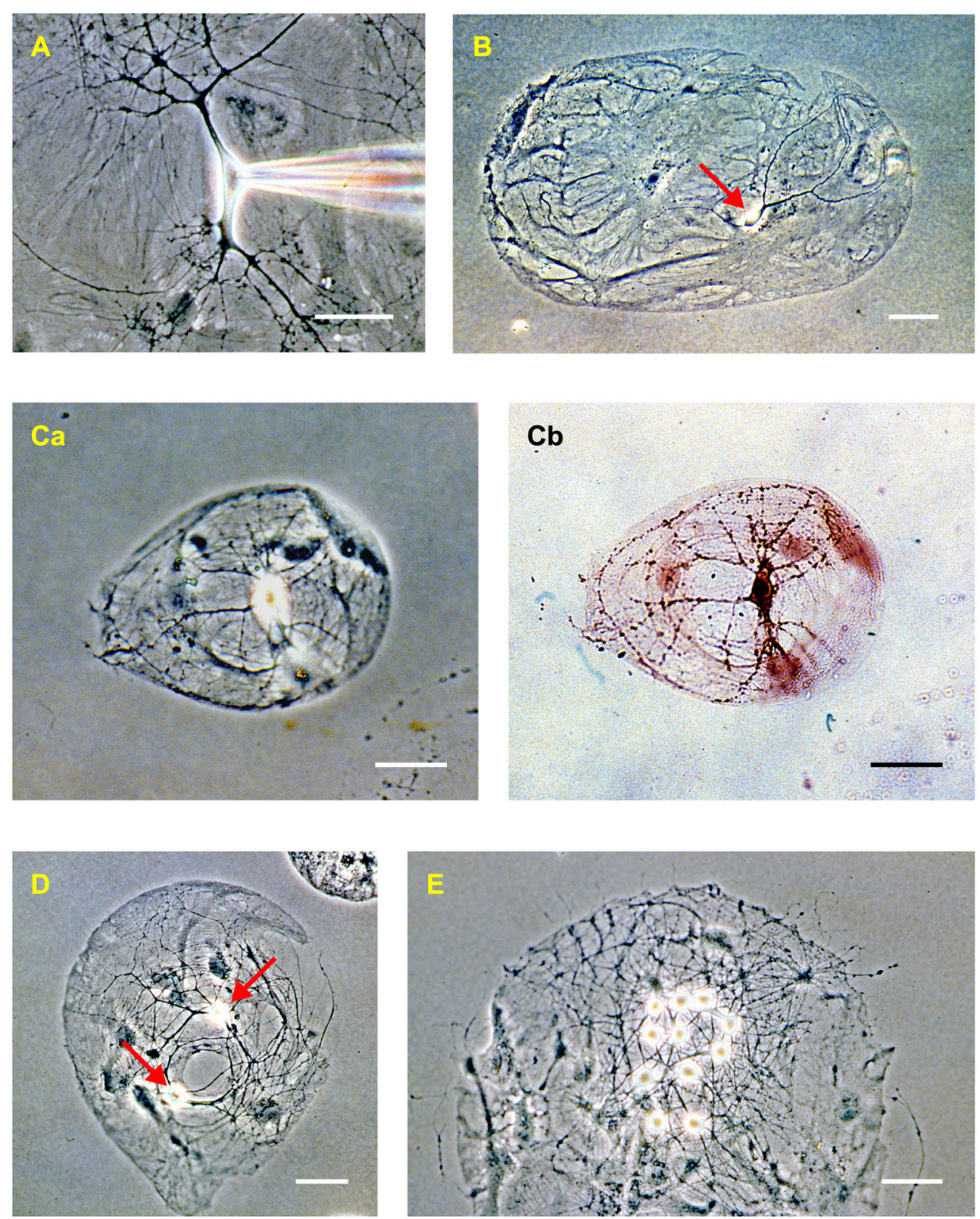

FIGURE 2 | Rat hippocampal neurons in dissociated culture (2-3 weeks in vitro). (A) Phase-contrast image of an isolated neuron in a mass culture showing the phase-bright soma, thick tapering dendrites, and thin untapered axons. Confluent astrocytes are visible beneath the neuron. A patch electrode, entering from the right, is positioned on the soma. (B) "Microdot" or "microisland" of astrocytes occupied by a single neuron (red arrow). This is a comparatively large island. (C) A microisland of more typical size, occupied by a single neuron imaged using phase contrast $\mathbf{( C a )}$ and bright-field $\mathbf{( C b )}$ microscopy after processing with a synapsin antibody to reveal autapses (dark spots on the processes in Cb). (D) Example of a microisland occupied by two neurons (red arrows). (E) Example of a microisland with multiple neurons. The scale bar in all panels is $50 \mu \mathrm{m}$.

If the neurons still die on plastic, they may require an astrocyte feeder layer, particularly if using mouse tissue or tissue from older (e.g., > P5) animals. Ideally, the astrocyte feeder layer should form a homogeneous extent of flat cells without too many gaps or overgrowth (e.g., Figure 2A). This is normally achieved by treating with an antimitotic agent (e.g., araC) as they approach confluence. The astrocyte cultures should also not be too old. We find that $<3-4$ weeks is preferred. Finally, the culture medium is probably the most important determinant of healthy cultures. Commercially available formulations are constantly evolving and some experimentation may be required to find the optimum for your application. Generally speaking, the serum-containing medium is better for growing astrocytes but it may be 
compromised by unpredictable variability between different lots of serum. Serum-free medium has the advantage of greater consistency but not all cell types may thrive in the absence of serum. Whatever the choice of medium, a universal precaution is that it should be fresh, sterile and adjusted to the correct $\mathrm{pH}$ and osmolarity.

\section{ELECTROPHYSIOLOGICAL RECORDINGS FROM AUTAPSES}

In this section, I will give tips for performing the most basic kind of electrophysiology experiment with autaptic cultures, i.e., a whole-cell patch-clamp recording of autaptic current from a single, isolated neuron under physiological conditions. These techniques are readily extended to more elaborate experiments of the kind that are summarized in later sections.

\section{Choosing a Neuron}

Broadly speaking, cultures can be used for electrophysiology experiments after $\sim 7$ days in vitro (DIV), although autaptic currents are expected to be small at that stage (Gomperts et al., 2000). Longer time in culture typically leads to a greater number of autaptic connections and larger currents per neuron (Gomperts et al., 2000), but there is also a progressive loss of neurons with culture age. We find that $\sim 14-21$ DIV is a good compromise for measuring large autaptic currents from healthy rodent neurons.

It is common to perform autaptic culture experiments in a HEPES-buffered bath solution at room temperature without bubbling or perfusion (e.g., Bekkers, 2005). Other standard bath solutions could also be used, for example, a perfused, carbogen-bubbled, bicarbonate-buffered bath solution warmed to physiological temperature, as in slice experiments (e.g., Ikeda et al., 2018). A phase-contrast microscope provides the best optics for observing neurites in culture (Figure 2). This may be important if one wishes to ensure, for example, that axons are not crossing the space between microislands. However, other types of contrast enhancement, such as differential interference contrast (DIC) or Hoffman modulation contrast, are acceptable for most studies with autaptic cultures.

After placing a whole coverslip in the chamber, the entire coverslip should be scanned at low magnification (e.g., $10 \times$ objective) to find potential microislands occupied by a single neuron. The shapes and sizes of these islands can be very diverse (Figure 2). In many cases it is unambiguous that a single neuron is present; in other cases, the identification is less clear. For example, glial overgrowth may obscure the view, or the soma of a glial cell (e.g., an oligodendrocyte) might be mistaken for that of a neuron. At other times the putative single neuron may occupy a very small island and it can be difficult to know if another neuron is hidden underneath the first. In these cases, the electrophysiology must be called upon to provide a definitive answer (see next section).

Identification of the type of neuron (e.g., glutamatergic, GABAergic, subtypes of these) can be inconclusive if simply based upon morphology. Dendritic morphology is often altered in culture, and in any case, the confinement of a neuron to a small island can make its dendrites hard to see. The best strategy is to prepare cultures from transgenic tissue in which the neurons of interest are fluorescently labeled (e.g., Ikeda et al., 2008).

During this initial scan, it is important to establish the health of the culture. The underlying layer of astrocytes should uniformly cover the islands of the permissive substrate, and the larger islands on the plate should be occupied by significant numbers of healthy-looking neurons (e.g., Figure 2E). There should be no sign of detachment of the astrocytes from the substrate, or of the agarose coating (which looks like a thin, transparent membrane) from the glass. If the culture contains clumps of many neuronal somata, perhaps extending thick fascicles of neurites between the clumps, then the plating density was too high. If there are healthy astrocytes but few neurons, then the plating density may have been too low.

Incidentally, cultures sometimes contain cells with vigorously waving cilia. These are ependymal cells from the meninges. They do not seem to interfere with experiments.

\section{Whole-Cell Patch-Clamp}

Standard patch-clamp techniques can be used with autaptic cultures, with a few precautions that are discussed below. After obtaining a stable whole-cell recording from the soma of an isolated neuron, the cell is voltage-clamped at a hyperpolarized holding potential (e.g., $-60 \mathrm{mV}$ ) and a $1 \mathrm{~ms}$-long depolarizing voltage-clamp step to $0 \mathrm{mV}$ is applied (Figure $\mathbf{3 A}$; this shows a hyperpolarizing test pulse followed by two depolarizing steps in succession). Each depolarizing step produces, in sequence, an outward capacitance transient (labeled 1 in Figure 3B), an inward "action current" (unclamped action potential; 2 in Figure 3B) and an inward capacitance transient (3 in Figure 3B). The action current escapes into the axon as a propagating action potential and, if autapses are present, a voltage-clamped autaptic current appears after a delay ( 4 in Figure 3B). In this example the neuron is glutamatergic and a fast inward excitatory autaptic current is observed. This autaptic current is selectively blocked by an inhibitor of AMPA receptors (Figure 3B, cyan trace). A similar experiment with an isolated GABAergic neuron shows an inhibitory autaptic current with slower kinetics, as expected for a current mediated by $\mathrm{GABA}_{\mathrm{A}}$ receptors (Figure 3C; in this example the pipette solution contained high $\mathrm{Cl}^{-}$).

Experiments can also be performed in current-clamp mode, in which case autaptic potentials would be recorded (Bekkers and Stevens, 1991). However, voltage-clamp recordings are generally more convenient because the repeated firing of self-exciting neurons can generally be more effectively controlled; thus, most investigators use voltage clamp.

This basic autapse experiment illustrates some precautions to keep in mind when measuring autaptic currents. First, the internal solution should allow the neuron to fire brief action potentials, i.e., it should typically be a high- $\mathrm{K}^{+}$solution to enable rapid spike repolarization. This is necessary to ensure the presynaptic action potential and the postsynaptic autaptic response are temporally dissociated, given that both are recorded in the same electrode (Figure 3). An internal solution that blocks $\mathrm{K}^{+}$channels, such as a high-Cs ${ }^{+}$solution of the kind commonly 


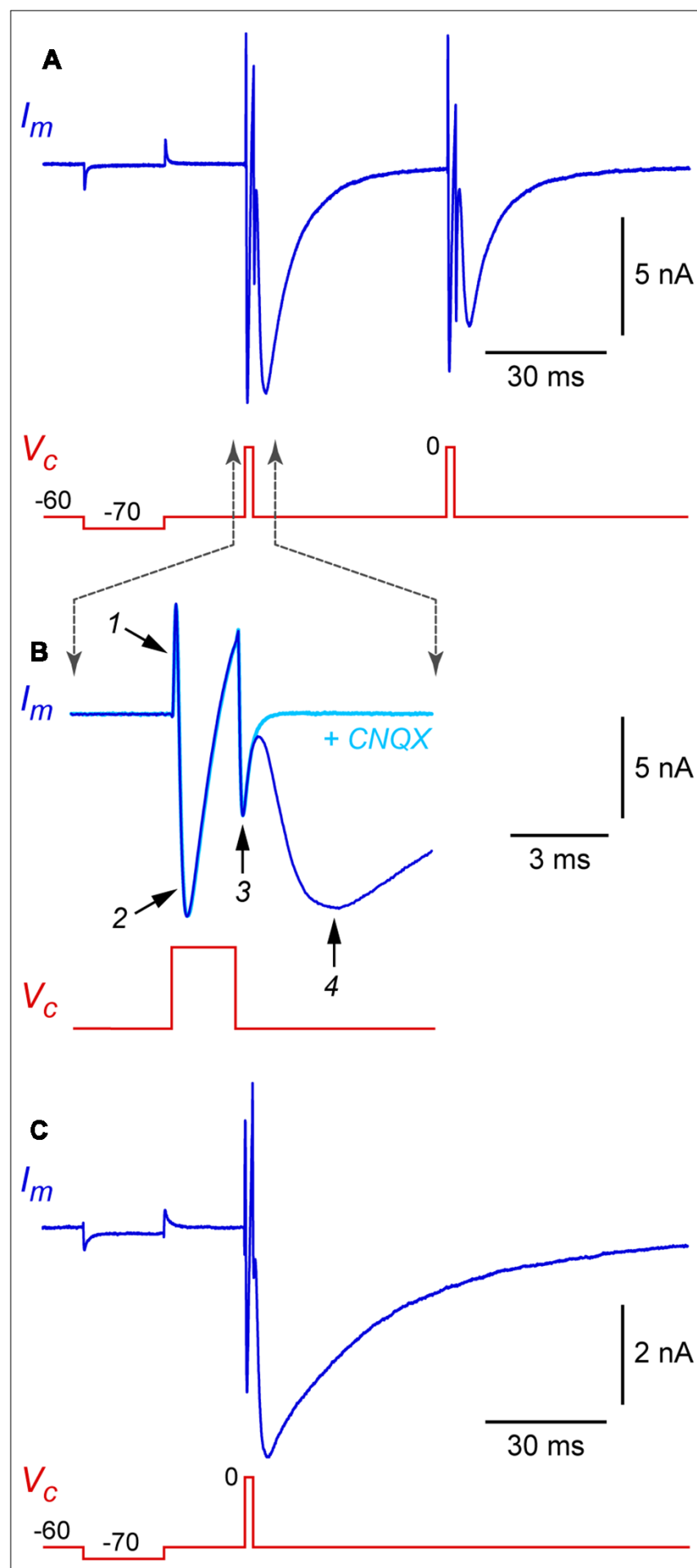

FIGURE 3 | Example autaptic postsynaptic currents recorded from isolated rat hippocampal neurons. (A) Membrane current $\left(I_{m}\right.$, blue trace) recorded in response to a voltage clamp command $\left(V_{C}\right.$, red trace) in an isolated glutamatergic neuron. In this example, two depolarizing stimuli (each a 2 $\mathrm{ms}$-long step from $-60 \mathrm{mV}$ holding potential to $0 \mathrm{mV}$ ) were applied at a $50 \mathrm{~ms}$ interval. A hyperpolarizing test pulse (20 ms-long step from -60 to $-70 \mathrm{mV}$ ) preceded the depolarizing stimuli. (B) Same data shown on an expanded time scale to illustrate the phases of the $I_{m}$ response. Also shown

(Continued)
FIGURE 3 | Continued

(cyan trace) is $I_{m}$ from the same cell immediately after switching to a flow pipe containing $20 \mu \mathrm{M}$ CNQX (6-cyano-7-nitroquinoxaline-2,3-dione) to block the excitatory postsynaptic current (EPSC). Labeled traces are (1) "on" capacitance transient, (2) action current ( $I_{\mathrm{Na}}$ and $I_{K}$ ), (3) "off" capacitance transient, and (4) autaptic EPSC. (C) Similar recording from an isolated GABAergic interneuron, showing an autaptic inhibitory postsynaptic current (autaptic IPSC) in response to a single stimulus. Same time scale as in panel (A); note the slower kinetics. In this experiment the pipette solution contained high $\mathrm{Cl}^{-}$, giving an inward current at $-60 \mathrm{mV}$ holding potential. Experiments in this figure were done at room temperature without series resistance compensation.

chosen to improve voltage clamp at depolarized potentials, should not be used.

A second precaution is that care must be taken to minimize voltage clamp errors. Autaptic currents can be large (up to $10 \mathrm{nA}$ ) and voltage escape could lead to uncontrolled action potentials and a reverberating feedback response in the neuron (Segal and Furshpan, 1990; Segal, 1991). Lower-resistance patch electrodes should generally be selected (e.g., <3-4 M $\Omega$ ) to facilitate the achievement of low $(<10-15 \mathrm{M} \Omega)$ electrode series resistance $\left(R_{S}\right)$. In addition to improving voltage control, low $R_{s}$ enables fast whole-cell capacitance transients (labeled 1 and 3 in Figure $3 \mathbf{B}$ ) which are less likely to merge with and obscure the closely following autaptic current. If merging does occur and is a concern, a workaround is to pharmacologically isolate the transients and action current by briefly superfusing a blocker of postsynaptic receptors (e.g., CNQX for glutamatergic autapses; Figure 3B, cyan trace), then digitally subtracting the blocked trace from the unblocked trace. This procedure should yield the pure autaptic current with minimal contamination from transients. In any case, electronic series resistance compensation should generally be used when measuring autaptic currents to minimize voltage clamp errors (Williams, 2004). Some investigators further reduce voltage clamp errors by applying a low concentration of blocker of postsynaptic receptors to reduce the amplitude of the autaptic current (Otsu et al., 2004).

A related precaution is to ensure that $R_{s}$ remains as stable as possible throughout the experiment. It is common for $R_{s}$ to increase steadily over time, which destabilizes the $R_{s}$ compensation and causes an apparent rundown in the amplitude of the autaptic current. We have found that this increase in $R_{s}$ can be much reduced by using internal solutions with a higher osmolarity (e.g., $330 \mathrm{mOsm} / \mathrm{kg}$ cf $310 \mathrm{mOsm} / \mathrm{kg}$ for the external solution; Bekkers, 2005). Higher internal osmolarity causes the neuron to swell slightly, possibly reducing a tendency of intracellular organelles to block the electrode tip.

Finally, autaptic currents sometimes exhibit delayed peaks without a preceding action current. This is often a sign that there is more than one neuron on the microdot, giving rise to a polysynaptic circuit. For some kinds of experiments, the delayed polysynaptic currents may not be a concern, particularly if the early autaptic current dominates. Another strategy, if the second neuron is visible, is to destroy the first neuron using the patch electrode (e.g., by aspirating the soma) then lower a new electrode 
to record from the second neuron. This strategy is only useful if the presence on the second neuron of remnant synapses from the first neuron is not a concern.

\section{APPLICATIONS OF AUTAPTIC CULTURES}

In this section, I will illustrate the utility of autaptic cultures by giving an overview of studies that have made use of them over the past three decades. I will organize my survey to highlight the unique technical advantages of autapses while paying less attention to the specific research questions being addressed. Most of the examples will be electrophysiology experiments using hippocampal and neocortical cultures, but some use autaptic cultures from other brain areas, including the thalamus (Moechars et al., 2006), basal forebrain (Allen, 2006), nucleus accumbens (Shi and Rayport, 1994), raphé nucleus (Johnson, 1994) and ventral tegmental area (Sulzer et al., 1998; Michel and Trudeau, 2000). First, I will make general comments about the advantages and disadvantages of autaptic cultures.

\section{Advantages}

Like any dissociated culture model, autaptic cultures provide the convenience of a simplified two-dimensional structure: easy access to individual cells for making electrical recordings, and excellent visibility for imaging experiments. Also, however, autaptic cultures offer unique advantages (Figure 4).

(1) The convenience of recording an unambiguous monosynaptic connection with a single electrode (Figure 4A). Because of the ease of recording, autaptic cultures provide a simple assay system for studying factors that determine synaptic strength.

(2) The ability to study a single axon and all its synaptic connections (Figure 4A). A single axon means that the
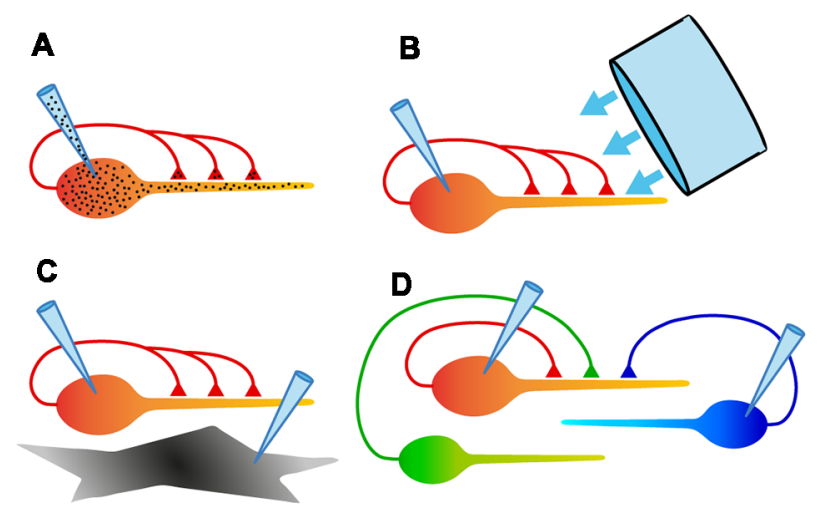

FIGURE 4 | Schematic diagrams summarizing some of the experimental configurations possible with autaptic cultures. (A) Electrical recording from assured monosynaptic connections in a homogeneous set of autapses using a single electrode, and control of both pre- and postsynaptic intracellular solutions using a single electrode. (B) Ability to rapidly and uniformly apply extracellular solutions or light stimulation to all autaptic connections received by that neuron. (C) Study of signaling between a neuron and an underlying astrocyte, using two recording electrodes. (D) Study of competition between two or more neurons confined to a microisland. This also allows simultaneous comparison of synaptic and autaptic responses in the same neuron. properties of a defined population of synaptic boutons can be studied, with no ambiguity about the origin of evoked or spontaneous synaptic events.

(3) Access to a homogeneous population of modified synapses (Figure 4A). Genetic modification of an isolated neuron ensures that all inputs onto the neuron are modified identically, providing an ideal testbed for structure-function studies.

(4) Complete control over the extracellular environment of a neuron and all its synapses (Figure 4B). Because all of their processes are restricted to a small area, autaptic cultures allow the rapid, homogeneous application of solutions or optical stimuli to an entire neuron, facilitating useful assays.

(5) The ability to define the neighborhood of a neuron and study intercellular interactions (Figures 4C,D). By selecting microislands occupied by different types or numbers of neurons or glia, interactions between these cell types can be explored in a simple system.

Examples of studies that explicitly draw upon these advantages are given in later sections.

\section{Disadvantages}

Autaptic cultures are susceptible to disadvantages that apply to culture models in general. For instance, cultured neurons commonly lose the dendritic and axonal morphologies that characterize their appearance in vivo. Their intrinsic functional properties might also be modified, and this must be checked or stated as a proviso (see next section). In addition to these general concerns about cultures, however, autaptic cultures have some specific disadvantages. Because the same neuron provides both the presynaptic and postsynaptic compartment, it may be impossible to separate processes that occur both preand postsynaptically. For example, $\mathrm{Ca}^{2+}$ signals that originate from the presynaptic action potential and the postsynaptic response may be entangled because of dendritic backpropagation (Otsu and Murphy, 2004). It is also difficult to depolarize the postsynaptic membrane without affecting presynaptic excitability (Bekkers and Stevens, 1991). When performing whole-cell patch-clamp experiments, one must be aware of the possibility of "washout" of key intracellular components, which might be a greater concern for autaptic cultures because both preand postsynaptic components could be vulnerable. That said, stable autaptic responses can generally be obtained, provided the electrode series resistance and other recording conditions are carefully controled (see "Whole-Cell Patch-Clamp" section). Lastly, the setting up of autaptic cultures is more fickle and labor intensive than that of conventional cell cultures, chiefly because more elaborate substrate preparation is required.

\section{Comparison of Autapses With Synapses in Other Model Systems}

As a prelude to discussing the applications of autaptic cultures, I will briefly comment on comparisons between the electrical properties of autapses and synapses in two other widely-used model systems, mass cultures, and acute brain slices. 


\section{Comparison With Interneuronal Synapses in Mass Cultures}

The basic biophysical features of autapses and synapses in mass cultures are reported to be the same (Bekkers and Stevens, 1991) or similar (Shi and Rayport, 1994; Mennerick et al., 1995; Liu et al., 2009, 2013). Autaptic EPSCs have somewhat larger amplitudes and readily-releasable vesicle pool sizes than their mass-culture counterparts (Mennerick et al., 1995; Liu et al., 2013), and differences in short-term plasticity have also been reported (Liu et al., 2013). Overall, however, autapses and interneuronal synapses in culture appear to function similarly.

\section{Comparison With Synapses in Acute Slices}

Dissociated cultures-including autaptic cultures-are less able to preserve the synaptic "microenvironment" than are more intact preparations, like acute slices, and this raises questions about the wider applicability of findings made in the culture setting. Nevertheless, many experimental findings made with autapses have translated well to the intact brain. For example, detailed molecular studies of the synaptic vesicle cycle at autapses (see "A Homogeneous Population of Modified Synapses" section) have been confirmed more generally (Chamberland and Tóth, 2016; Dittman and Ryan, 2019). Certain types of synaptic plasticity that have been studied in slices are also faithfully replicated in autaptic cultures. For instance, depolarizationinduced suppression of excitation (DSE; Straiker and Mackie, 2009) and inhibition (DSI; Straiker and Mackie, 2005; Kellogg et al., 2009) are present at autapses, as is long-term depression (LTD; Goda and Stevens, 1996; Tong et al., 1996; Kumura et al., 2000). Cultured dentate granule cells form autapses that resemble mossy fiber inputs to CA3 pyramidal cells, including expression of a presynaptic form of long-term potentiation (Rost et al., 2010). On the other hand, studies have also highlighted the potential risks of using cultures. For example, reports on the co-release of neurotransmitters (Hnasko and Edwards, 2012) and the efficacy of neuromodulators (Bekkers et al., 1996) show that the exact experimental conditions in culture can have an impact on the findings. All of this reinforces the point that, as for any model system, effective use of autaptic cultures must balance convenience and caution.

I will now turn to a survey of applications of autaptic cultures, organized under the five major advantages listed above in the "Advantages" section.

\section{Examples of Applications}

\section{The Convenience of a Single Electrode}

By providing access to large, monosynaptic currents that can be stably measured with a single electrode, autaptic cultures have been used in many key experiments on the biophysics of synaptic transmission. Early examples include studies of multivesicular release at glutamatergic synapses (Tong and Jahr, 1994b), how transporters shape neurotransmission (Tong and Jahr, 1994a; Diamond and Jahr, 1997), and how the kinetics of EPSCs are determined by asynchronous vesicle release and temperature (Diamond and Jahr, 1995; Pyott and Rosenmund, 2002). The role of presynaptic $\mathrm{Ca}^{2+}$ in determining neurotransmission has also been examined using autapses (Reid et al., 1998; Lee et al., 2000; Ikeda et al., 2008).

A different application has been to use autaptic cultures to study neuromodulation, again taking advantage of their convenience as an assay system. Modulators that have been characterized with autapses include histamine (Bekkers, 1993), adrenoceptor agonists (Raman et al., 1996), calcineurin (Tong et al., 1995), somatostatin (Boehm and Betz, 1997), clozapine (Michel and Trudeau, 2000), cyclic AMP (Gekel and Neher, 2008), amyloid $\alpha$ protein (Ripoli et al., 2013) and GABA acting via $\mathrm{GABA}_{\mathrm{B}}$ receptors (Valente et al., 2016). Several reports have also used autaptic cultures to examine short-term synaptic plasticity and its modulation (Mennerick and Zorumski, 1995a; Brody and Yue, 2000; Straiker and Mackie, 2007; Straiker et al., 2018). Lastly, autapses have been used as a convenient testbed for developing new molecular tools for the study of synaptic function (e.g., pHoenix, Rost et al., 2015).

\section{A Single Axon}

An autaptic neuron in culture is innervated by a single afferent (from itself) and thus provides access to a uniquely homogeneous population of presynaptic terminals-reliably stimulated-that can be used for the study of neurotransmitter release (Otsu and Murphy, 2004). For example, autaptic cultures have been utilized to measure the nonuniform probability of glutamate release from different boutons on the same axon (Rosenmund et al., 1993), the variable distribution of presynaptic $\mathrm{Ca}$ channel subtypes on a single afferent (Reid et al., 1997), and the differential invasion of glutamate-releasing terminals by action potentials (Prakriya and Mennerick, 2000). Autapses are also convenient for studying the synaptic vesicle cycle, for example, by using pharmacological approaches to count the number of releasable vesicles (Ikeda and Bekkers, 2009) or optical methods based on the styryl dyes (e.g., FM1-43) to follow vesicles through the cycle (Murthy et al., 1997; Murthy and Stevens, 1998). Experiments that load "false" neurotransmitters into synaptic vesicles have also been used to probe vesicle dynamics at autapses (Pan et al., 1993; Bekkers, 2005).

Another kind of experiment that relies on having a single axon takes advantage of the fact that both evoked and spontaneous postsynaptic currents originate from the same population of autaptic terminals, removing the ambiguity that typifies other kinds of synaptic circuits. For example, autaptic cultures been used to identify presynaptically silent synapses that give spontaneous but not evoked transmitter release (Kimura et al., 1997), and postsynaptically silent synapses that express only NMDA receptors (Gomperts et al., 1998). Autapses also allow the unambiguous study of phasic and asynchronous release from the same population of boutons (Otsu et al., 2004; Chang and Mennerick, 2010).

Lastly, the presence of a single axon enables definitive experiments showing the co-release of neurotransmitters from one neuron. For instance, autaptic cultures have been used to demonstrate the co-release of glutamate and serotonin from raphé neurons (Johnson, 1994) and of glutamate 
and dopamine from neurons in the ventral tegmental area (Sulzer et al., 1998).

\section{A Homogeneous Population of Modified Synapses}

Perhaps the most widely used application of autaptic cultures has been in structure-function studies, which take advantage of the ability to genetically modify a single isolated neuron and to examine the consequences of that modification for synaptic transmission, secure in the knowledge that all autapses received by that neuron are identically modified. The use of this culture system also enables the study of mutations that are postnatal lethal.

Genetically-modified autaptic cultures have been used to answer fundamental questions about the SNARE proteins, including SNAP-25 and SNAP-23 (Delgado-Martínez et al., 2007; Weber et al., 2014), syntaxin-1 (Gerber et al., 2008) and synaptobrevin-1 (Young, 2005; Zimmermann et al., 2014). Key experiments on the presynaptic $\mathrm{Ca}^{2+}$ sensor, synaptotagmin, have also been performed using autapses. For example, mutational analyses of synaptotagmin-1 (Fernández-Chacón et al., 2001; Stevens and Sullivan, 2003; Han et al., 2004; Nishiki and Augustine, 2004; Schupp et al., 2016; Chang et al., 2018) and synaptotagmin-4 (Ting et al., 2006) have elaborated details of the $\mathrm{Ca}^{2+}$ binding and synaptic vesicle fusion processes. The roles of many other presynaptic auxiliary proteins have been explored at autapses, including Munc13 (Augustin et al., 1999; Rosenmund et al., 2002; Varoqueaux et al., 2002; Junge et al., 2004; Basu et al., 2007), Munc18 (Meijer et al., 2015), synapsin (Gitler et al., 2004, 2008; Baldelli et al., 2007), bassoon (Altrock et al., 2003), synaptic vesicle protein 2 (Custer et al., 2006), RIM1 $\alpha$ (Calakos et al., 2004), snapin (Thakur et al., 2004) and Rab3 (Schlüter et al., 2004). A number of these experiments took advantage of another convenient feature of autaptic cultures - the ability to completely superfuse a single neuron-to look for changes in the size of the readily-releasable pool (RRP) of synaptic vesicles. This feature will be further discussed in the next section.

Other researchers have used autapses and molecular genetic approaches to study the involvement of vesicular glutamate transporters in synaptic transmission (Fremeau et al., 2004; Wojcik et al., 2004; Zimmermann et al., 2015), as well as the properties of presynaptic voltage-gated $\mathrm{Ca}^{2+}$ channels (Few et al., 2012; Nanou et al., 2016). Lastly, a variety of disease models have been explored using this strategy. For example, genetic models of Alzheimer's disease (Pratt et al., 2011), autism (Weston et al., 2014) and epilepsy (Heeroma et al., 2009) have been implemented and studied in autaptic cultures.

\section{Control Over the Extracellular Environment}

The ability to uniformly perfuse extracellular solution over an isolated autaptic neuron brings obvious advantages in pharmacological experiments, like the neuromodulation studies mentioned earlier ("The Convenience of a Single Electrode" section). However, uniform perfusion also enables other kinds of experiments that are difficult or impossible to achieve using other systems. Two main types of such experiments have been performed: measurements of synaptic vesicle pools using the application of hypertonic sucrose solution, and studies that disentangle subsynaptic (strictly, "sub-autaptic") and extrasynaptic receptors by exploiting the unique configuration of autaptic cultures.

Sucrose experiments depend on the classical finding that hypertonic solution causes the direct release of synaptic vesicles without electrical stimulation (Fatt and Katz, 1952). By applying pulses of sucrose solution to the entire dendritic arbor of an isolated neuron on a small island, all presynaptic vesicle pools can be identically stimulated with a hypertonic challenge and the resulting postsynaptic electrical response can be recorded at the soma. Critically, spontaneous or electrically-evoked release from those same pools can also be recorded, allowing a comparison between the postsynaptic responses to the different release mechanisms. For example, by measuring the total charge carried by the (multi-vesicle) sucrose-evoked response, then dividing this by the mean charge of the (single-vesicle) spontaneous "miniature" synaptic response measured in the same neuron, one can estimate the total size of the RRP of synaptic vesicles across all release sites on that neuron (Rosenmund and Stevens, 1996; Stevens and Williams, 2007).

This method has been widely used to measure changes in the RRP size following genetic mutations, as mentioned in the previous section ("A Homogeneous Population of Modified Synapses"). The approach has also been used to study changes in RRP size following different kinds of plasticity, for example, long-term depression (Goda and Stevens, 1998), short-term depression (Stevens and Wesseling, 1998, 1999b) and augmentation (Stevens and Wesseling, 1999a; Garcia-Perez and Wesseling, 2008), as well as other factors that alter the RRP size at autapses (Stevens and Sullivan, 1998; Moulder et al., 2004; Moulder and Mennerick, 2005).

The second main type of experiment that exploits the ease of autapse superfusion is a method for distinguishing subsynaptic and extrasynaptic NMDA receptors. This experiment depends on the fact that electrical stimulation (e.g., via a whole-cell electrode at the soma) selectively activates subsynaptic NMDA receptors, whereas superfusing the entire neuron with glutamate activates both sub- and extrasynaptic NMDA receptors. Hence, by comparing these two responses one can infer the distinct properties of both pools of NMDA receptors (Tovar and Westbrook, 1999). In an elegant variation on this experiment, subsynaptic NMDA receptors are "tagged" by blocking them with an activity-dependent antagonist (MK801) while electrically stimulating at the soma; the subsequent response to whole-cell application of glutamate must then be entirely due to unblocked extrasynaptic NMDA receptors (Tovar and Westbrook, 2002).

This approach has been used in several interesting studies. For example, the mobility and turnover of the different pools of NMDA receptors have been measured (Li et al., 2002; Tovar and Westbrook, 2002), as well as their subunit composition (Tovar and Westbrook, 1999; Thomas et al., 2006), pharmacology (Xia et al., 2010; Vyklicky et al., 2016) and desensitization properties (Li et al., 2003).

Lastly, the compact dendritic arbor of autaptic cultures facilitates the use of optical techniques for the study of synaptic function. For example, autaptic cultures permit the uniform 
elevation of intracellular $\mathrm{Ca}^{2+}$ in calcium uncaging experiments (Burgalossi et al., 2012). However, optogenetic techniques have not been used with autaptic cultures and provide an opportunity for interesting experiments in the future.

\section{A Defined Neighborhood}

The applications described so far have highlighted the isolation of autaptic neurons, but this final application takes advantage of microislands as a useful model for studying intercellular interactions in the local neighborhood. These experiments fall into two broad classes: signaling between an autaptic neuron and the underlying glial cells, and competition between neurons on microislands occupied by two or more neurons.

Neuron/glia signaling has been studied by making dual whole-cell recordings from an isolated autaptic neuron and an underlying glial cell (Mennerick and Zorumski, 1994, 1995a; Mennerick et al., 1996). These experiments have shown, for example, how transporters in the glia modulate the timecourse of the autaptic EPSC in the neuron (Mennerick and Zorumski, 1995b; Mennerick et al., 1999), and how autaptic neurons grown without glial contact exhibit more asynchronous neurotransmitter release (Sobieski et al., 2015). Culturing without contact with glia has also been shown to reduce the number of autapses that form (Ullian et al., 2001).

For studying interactions between neurons, microislands with two or more neurons are selected (Figure 4D). Early work used microislands with small numbers of neurons to study epileptiform activity, which tends to be very strong when neurons are spatially constrained in this way (Segal and Furshpan, 1990; Segal, 1991, 1994). More recent work, however, has tended to focus on two-neuron microislands for "mix and match" experiments. For example, it has been shown that chronically silencing one of a pair of isolated excitatory neurons leads to characteristic changes in the postsynaptic AMPA receptor subunit composition in each neuron (Harms et al., 2005). Other work has explored the competition between glutamatergic and GABAergic neurons when grown as mixed or homotypic pairs (Rao et al., 2000; Chang et al., 2014; Wierda and Sørensen, 2014), or between wildtype and mutant neurons that lack presynaptic proteins involved in transmitter release (Tarsa and Goda, 2002; García-Pérez et al., 2015). Finally, two-cell microislands with different combinations of striatal, cortical and thalamic neurons have been used to examine the effect of

\section{REFERENCES}

Allen, T. G. (2006). Preparation and maintenance of single-cell micro-island cultures of basal forebrain neurons. Nat. Protoc. 1, 2543-2550. doi: 10.1038/ nprot.2006.394

Altrock, W. D., tom Dieck, S., Sokolov, M., Meyer, A. C., Sigler, A., Brakebusch, C., et al. (2003). Functional inactivation of a fraction of excitatory synapses in mice deficient for the active zone protein bassoon. Neuron 37, 787-800. doi: 10.1016/s0896-6273(03)00088-6

Augustin, I., Rosenmund, C., Südhof, T. C., and Brose, N. (1999). Munc13-1 is essential for fusion competence of glutamatergic synaptic vesicles. Nature 400, 457-461. doi: 10.1038/22768 glutamatergic input on refining striatal output (Paraskevopoulou et al., 2019).

\section{CONCLUSION}

Autaptic cultures provide the ultimate in synaptic simplicity: a one-neuron circuit. Despite a current tendency to study complex and preferably in vivo neural networks, autaptic cultures still have an important place in the toolbox of cellular neuroscientists because they enable straightforward, elegant experiments that address fundamental questions about synaptic transmission. The examples of applications given in this review provide a taste of the diversity of experiments that are possible with autaptic cultures, and will hopefully inspire novel applications, perhaps involving new-generation optical technologies. Of course, like all model systems, autaptic cultures have advantages and disadvantages, and these must be carefully weighed up when deciding whether autapses are the right tool for the job. It is hoped that this review has provided enough information to make that decision easier, and will assist in the implementation of autaptic culture experiments with the minimum pain and maximum prospect of success.

\section{DATA AVAILABILITY STATEMENT}

The datasets generated for this study are available on request to the corresponding author.

\section{AUTHOR CONTRIBUTIONS}

JB wrote the manuscript and prepared the figures.

\section{FUNDING}

This work was supported by Project Grant 1128320 from the National Health and Medical Research Council of Australia and Discovery Project Grant DP 180101034 from the Australian Research Council.

\section{ACKNOWLEDGMENTS}

I thank Jane Sullivan for comments on the manuscript. I also thank the reviewers for helpful suggestions.

Bacci, A., and Huguenard, J. R. (2006). Enhancement of spike-timing precision by autaptic transmission in neocortical inhibitory interneurons. Neuron 49, 119-130. doi: 10.1016/j.neuron.2005.12.014

Baldelli, P., Fassio, A., Valtorta, F., and Benfenati, F. (2007). Lack of synapsin I reduces the readily releasable pool of synaptic vesicles at central inhibitory synapses. J. Neurosci. 27, 13520-13531. doi: 10.1523/JNEUROSCI.315107.2007

Basu, J., Betz, A., Brose, N., and Rosenmund, C. (2007). Munc13-1 C1 domain activation lowers the energy barrier for synaptic vesicle fusion. J. Neurosci. 27, 1200-1210. doi: 10.1523/JNEUROSCI.4908-06.2007

Bekkers, J. M. (1993). Enhancement by histamine of NMDA-mediated synaptic transmission in the hippocampus. Science 261, 104-106. doi: 10.1126/science. 8391168 
Bekkers, J. M. (1998). Are autapses prodigal synapses? Curr. Biol. 8, R52-R55. doi: 10.1016/s0960-9822(98)70033-8

Bekkers, J. M. (2003). Synaptic transmission: functional autapses in the cortex. Curr. Biol. 13, R433-R435. doi: 10.1016/s0960-9822(03)00363-4

Bekkers, J. M. (2005). Presynaptically silent GABA synapses in hippocampus. J. Neurosci. 25, 4031-4039. doi: 10.1523/JNEUROSCI.4969-04.2005

Bekkers, J. M. (2009). Synaptic transmission: excitatory autapses find a function? Curr. Biol. 19, R296-R298. doi: 10.1016/j.cub.2009.02.010

Bekkers, J. M., and Stevens, C. F. (1991). Excitatory and inhibitory autaptic currents in isolated hippocampal neurons maintained in cell culture. Proc. Natl. Acad. Sci. U S A 88, 7834-7838. doi: 10.1073/pnas.88.17.7834

Bekkers, J. M., Vidovic, M., and Ymer, S. (1996). Differential effects of histamine on the N-methyl-D-aspartate channel in hippocampal slices and cultures. Neuroscience 72, 669-677. doi: 10.1016/0306-4522(95)00586-2

Boehm, S., and Betz, H. (1997). Somatostatin inhibits excitatory transmission at rat hippocampal synapses via presynaptic receptors. J. Neurosci. 17, 4066-4075. doi: 10.1523/JNEUROSCI.17-11-04066.1997

Brewer, G. J. (1997). Isolation and culture of adult rat hippocampal neurons. J. Neurosci. Methods 71, 143-155. doi: 10.1016/s0165-0270(96)00136-7

Brewer, G. J., and Cotman, C. W. (1989). Survival and growth of hippocampal neurons in defined medium at low density: advantages of a sandwich culture technique or low oxygen. Brain Res. 494, 65-74. doi: 10.1016/00068993(89)90144-3

Brody, D. L., and Yue, D. T. (2000). Relief of G-protein inhibition of calcium channels and short-term synaptic facilitation in cultured hippocampal neurons. J. Neurosci. 20, 889-898. doi: 10.1523/JNEUROSCI.20-03-00889.2000

Brown, L. D., Kim, K.-M., Nakajima, Y., and Nakajima, S. (1993). The role of $\mathrm{G}$ protein in muscarinic depolarization near resting potential in cultured hippocampal neurons. Brain Res. 612, 200-209. doi: 10.1016/00068993(93)91661-b

Burgalossi, A., Jung, S., Man, K. N., Nair, R., Jockusch, W. J., Wojcik, S. M., et al. (2012). Analysis of neurotransmitter release mechanisms by photolysis of caged $\mathrm{Ca}^{2+}$ in an autaptic neuron culture system. Nat. Protoc. 7, 1351-1365. doi: 10.1038/nprot.2012.074

Calakos, N., Schoch, S., Sudhof, T. C., and Malenka, R. C. (2004). Multiple roles for the active zone protein RIM1 $\propto$ in late stages of neurotransmitter release. Neuron 42, 889-896. doi: 10.1016/j.neuron.2004.05.014

Chamberland, S., and Tóth, K. (2016). Functionally heterogeneous synaptic vesicle pools support diverse synaptic signalling. J. Physiol. 594, 825-835. doi: 10.1113/jp270194

Chang, C. L., Trimbuch, T., Chao, H. T., Jordan, J. C., Herman, M. A., and Rosenmund, C. (2014). Investigation of synapse formation and function in a glutamatergic-GABAergic two-neuron microcircuit. J. Neurosci. 34, 855-868. doi: 10.1523/JNEUROSCI.0229-13.2014

Chang, C. Y., and Mennerick, S. (2010). Dynamic modulation of phasic and asynchronous glutamate release in hippocampal synapses. J. Neurophysiol. 103, 392-401. doi: 10.1152/jn.00683.2009

Chang, S., Trimbuch, T., and Rosenmund, C. (2018). Synaptotagmin-1 drives synchronous $\mathrm{Ca}^{2+}$-triggered fusion by $\mathrm{C}_{2} \mathrm{~B}$-domain-mediated synapticvesicle-membrane attachment. Nat. Neurosci. 21, 33-40. doi: 10.1038/s41593017-0037-5

Cobb, S. R., Halasy, K., Vida, I., Nyiri, G., Tamás, G., Buhl, E. H., et al. (1997). Synaptic effects of identified interneurons innervating both interneurons and pyramidal cells in the rat hippocampus. Neuroscience 79, 629-648. doi: 10.1016/s0306-4522(97)00055-9

Connelly, W. M. (2014). Autaptic connections and synaptic depression constrain and promote $\gamma$ oscillations. PLoS One 9:e89995. doi: 10.1371/journal.pone. 0089995

Connelly, W. M., and Lees, G. (2010). Modulation and function of the autaptic connections of layer $\mathrm{V}$ fast spiking interneurons in the rat neocortex. J. Physiol. 588, 2047-2063. doi: 10.1113/jphysiol.2009.185199

Custer, K. L., Austin, N. S., Sullivan, J. M., and Bajjalieh, S. M. (2006). Synaptic vesicle protein 2 enhances release probability at quiescent synapses. J. Neurosci. 26, 1303-1313. doi: 10.1523/JNEUROSCI.2699-05.2006

Deleuze, C., Bhumbra, G. S., Pazienti, A., Lourenco, J., Mailhes, C., Aguirre, A., et al. (2019). Strong preference for autaptic self-connectivity of neocortical PV interneurons facilitates their tuning to $\gamma$-oscillations. PLoS Biol. 17:e3000419. doi: 10.1371/journal.pbio.3000419
Deleuze, C., Pazienti, A., and Bacci, A. (2014). Autaptic self-inhibition of cortical GABAergic neurons: synaptic narcissism or useful introspection? Curr. Opin. Neurobiol. 26, 64-71. doi: 10.1016/j.conb.2013.12.009

Delgado-Martínez, I., Nehring, R. B., and Sørensen, J. B. (2007). Differential abilities of SNAP-25 homologs to support neuronal function. J. Neurosci. 27, 9380-9391. doi: 10.1523/JNEUROSCI.5092-06.2007

Diamond, J. S., and Jahr, C. E. (1995). Asynchronous release of synaptic vesicles determines the time course of the AMPA receptor-mediated EPSC. Neuron 15, 1097-1107. doi: 10.1016/0896-6273(95)90098-5

Diamond, J. S., and Jahr, C. E. (1997). Transporters buffer synaptically released glutamate on a submillisecond time scale. J. Neurosci. 17, 4672-4687. doi: 10.1523/JNEUROSCI.17-12-04672.1997

Dittman, J. S., and Ryan, T. A. (2019). The control of release probability at nerve terminals. Nat. Rev. Neurosci. 20, 177-186. doi: 10.1038/s41583-0180111-3

Douglas, R. J., and Martin, K. A. (2007). Mapping the matrix: the ways of neocortex. Neuron 56, 226-238. doi: 10.1016/j.neuron.2007.10.017

Fasano, C., Thibault, D., and Trudeau, L. E. (2008). Culture of postnatal mesencephalic dopamine neurons on an astrocyte monolayer. Curr. Protoc. Neurosci. Chapter 3:Unit 3.21. doi: 10.1002/0471142301.ns0321s44

Fath, T., Ke, Y. D., Gunning, P., Götz, J., and Ittner, L. M. (2009). Primary support cultures of hippocampal and substantia nigra neurons. Nat. Protoc. 4, 78-85. doi: 10.1038/nprot.2008.199

Fatt, P., and Katz, B. (1952). Spontaneous subthreshold activity at motor nerve endings. J. Physiol. 117, 109-128.

Fenske, P., Grauel, M. K., Brockmann, M. M., Dorrn, A. L., Trimbuch, T., and Rosenmund, C. (2019). Autaptic cultures of human induced neurons as a versatile platform for studying synaptic function and neuronal morphology. Sci. Rep. 9:4890. doi: 10.1038/s41598-019-41259-1

Fernández-Chacón, R., Königstorfer, A., Gerber, S. H., García, J., Matos, M. F., Stevens, C. F., et al. (2001). Synaptotagmin I functions as a calcium regulator of release probability. Nature 410, 41-49. doi: 10.1038/35065004

Few, A. P., Nanou, E., Watari, H., Sullivan, J. M., Scheuer, T., and Catterall, W. A. (2012). Asynchronous $\mathrm{Ca}^{2+}$ current conducted by voltage-gated $\mathrm{Ca}^{2+}$ $\left(\mathrm{Ca}_{V}\right)-2.1$ and $\mathrm{Ca}_{V} 2.2$ channels and its implications for asynchronous neurotransmitter release. Proc. Natl. Acad. Sci. U S A 109, E452-E460. doi: 10.1073/pnas.1121103109

Fremeau, R. T. Jr., Kam, K., Qureshi, T., Johnson, J., Copenhagen, D. R., Storm-Mathisen, J., et al. (2004). Vesicular glutamate transporters 1 and 2 target to functionally distinct synaptic release sites. Science 304, 1815-1819. doi: 10.1126/science. 1097468

Furshpan, E. J., Landis, S. C., Matsumoto, S. G., and Potter, D. D. (1986). Synaptic functions in rat sympathetic neurons in microcultures. I. Secretion of norepinephrine and acetylcholine. J. Neurosci. 6, 1061-1079. doi: 10.1523/JNEUROSCI.06-04-01061.1986

Furshpan, E. J., MacLeish, P. R., O'Lague, P. H., and Potter, D. D. (1976). Chemical transmission between rat sympathetic neurons and cardiac myocytes developing in microcultures: evidence for cholinergic, adrenergic, and dual-function neurons. Proc. Natl. Acad. Sci. U S A 73, 4225-4229. doi: 10.1073/pnas.73.11.4225

García-Pérez, E., Mahfooz, K., Covita, J., Zandueta, A., and Wesseling, J. F. (2015). Levetiracetam accelerates the onset of supply rate depression in synaptic vesicle trafficking. Epilepsia 56, 535-545. doi: 10.1111/epi.12930

Garcia-Perez, E., and Wesseling, J. F. (2008). Augmentation controls the fast rebound from depression at excitatory hippocampal synapses. J. Neurophysiol. 99, 1770-1786. doi: 10.1152/jn.01348.2007

Gautam, V., Naureen, S., Shahid, N., Gao, Q., Wang, Y., Nisbet, D., et al. (2017). Engineering highly interconnected neuronal networks on nanowire scaffolds. Nano Lett. 17, 3369-3375. doi: 10.1021/acs.nanolett.6b05288

Gekel, I., and Neher, E. (2008). Application of an Epac activator enhances neurotransmitter release at excitatory central synapses. J. Neurosci. 28, 7991-8002. doi: 10.1523/JNEUROSCI.0268-08.2008

Gerber, S. H., Rah, J. C., Min, S. W., Liu, X., de Wit, H., Dulubova, I., et al. (2008). Conformational switch of syntaxin-1 controls synaptic vesicle fusion. Science 321, 1507-1510. doi: 10.1126/science.1163174

Gitler, D., Cheng, Q., Greengard, P., and Augustine, G. J. (2008). Synapsin IIa controls the reserve pool of glutamatergic synaptic vesicles. J. Neurosci. 28 , 10835-10843. doi: 10.1523/JNEUROSCI.0924-08.2008 
Gitler, D., Takagishi, Y., Feng, J., Ren, Y., Rodriguiz, R. M., Wetsel, W. C., et al. (2004). Different presynaptic roles of synapsins at excitatory and inhibitory synapses. J. Neurosci. 24, 11368-11380. doi: 10.1523/JNEUROSCI.379504.2004

Goda, Y., and Stevens, C. F. (1996). Long-term depression properties in a simple system. Neuron 16, 103-111. doi: 10.1016/s0896-6273(00)80027-6

Goda, Y., and Stevens, C. F. (1998). Readily releasable pool size changes associated with long term depression. Proc. Natl. Acad. Sci. U S A 95, 1283-1288. doi: $10.1073 /$ pnas.95.3.1283

Gomperts, S. N., Carroll, R., Malenka, R. C., and Nicoll, R. A. (2000). Distinct roles for ionotropic and metabotropic glutamate receptors in the maturation of excitatory synapses. J. Neurosci. 20, 2229-2237. doi: 10.1523/JNEUROSCI. 20-06-02229.2000

Gomperts, S. N., Rao, A., Craig, A. M., Malenka, R. C., and Nicoll, R. A. (1998). Postsynaptically silent synapses in single neuron cultures. Neuron 21, 1443-1451. doi: 10.1016/s0896-6273(00)80662-5

Goslin, K., Asmussen, H., and Banker, G. (1998). "Rat hippocampal neurons in low-density culture," in Culturing Nerve Cells, 2nd Edn. eds G. Banker and K. Goslin (Cambridge, MA: MIT Press), 339-370.

Guo, D., Wu, S., Chen, M., Perc, M., Zhang, Y., Ma, J., et al. (2016). Regulation of irregular neuronal firing by autaptic transmission. Sci. Rep. 6:26096. doi: $10.1038 /$ srep26096

Han, W., Rhee, J. S., Maximov, A., Lao, Y., Mashimo, T., Rosenmund, C., et al. (2004). N-glycosylation is essential for vesicular targeting of synaptotagmin 1. Neuron 41, 85-99. doi: 10.1016/s0896-6273(03)00820-1

Harms, K. J., Tovar, K. R., and Craig, A. M. (2005). Synapse-specific regulation of AMPA receptor subunit composition by activity. J. Neurosci. 25, 6379-6388. doi: 10.1523/JNEUROSCI.0302-05.2005

Heeroma, J. H., Henneberger, C., Rajakulendran, S., Hanna, M. G., Schorge, S., and Kullmann, D. M. (2009). Episodic ataxia type 1 mutations differentially affect neuronal excitability and transmitter release. Dis. Model. Mech. 2, 612-619. doi: $10.1242 / \mathrm{dmm} .003582$

Hnasko, T. S., and Edwards, R. H. (2012). Neurotransmitter corelease: mechanism and physiological role. Annu. Rev. Physiol. 74, 225-243. doi: 10.1146/annurevphysiol-020911-153315

Ikeda, K., and Bekkers, J. M. (2006). Autapses. Curr. Biol. 16:R308. doi: 10.1016/j. cub.2006.03.085

Ikeda, K., and Bekkers, J. M. (2009). Counting the number of releasable synaptic vesicles in a presynaptic terminal. Proc. Natl. Acad. Sci. U S A 106, 2945-2950. doi: 10.1073/pnas.0811017106

Ikeda, K., Suzuki, N., and Bekkers, J. M. (2018). Sodium and potassium conductances in principal neurons of the mouse piriform cortex: a quantitative description. J. Physiol. 596, 5397-5414. doi: 10.1113/jp275824

Ikeda, K., Yanagawa, Y., and Bekkers, J. M. (2008). Distinctive quantal properties of neurotransmission at excitatory and inhibitory autapses revealed using variance-mean analysis. J. Neurosci. 28, 13563-13573. doi: 10.1523/ JNEUROSCI.3350-08.2008

Ivenshitz, M., and Segal, M. (2010). Neuronal density determines network connectivity and spontaneous activity in cultured hippocampus. J. Neurophysiol. 104, 1052-1060. doi: 10.1152/jn.00914.2009

Jang, M. J., Kim, W. R., Joo, S., Ryu, J. R., Lee, E., Nam, Y., et al. (2016). Cell-type dependent effect of surface-patterned microdot arrays on neuronal growth. Front. Neurosci. 10:217. doi: 10.3389/fnins.2016.00217

Jiang, M., Yang, M., Yin, L., Zhang, X., and Shu, Y. (2015). Developmental reduction of asynchronous GABA release from neocortical fast-spiking neurons. Cereb. Cortex 25, 258-270. doi: 10.1093/cercor/bht236

Jiang, M., Zhu, J., Liu, Y., Yang, M., Tian, C., Jiang, S., et al. (2012). Enhancement of asynchronous release from fast-spiking interneuron in human and rat epileptic neocortex. PLoS Biol. 10:e1001324. doi: 10.1371/journal.pbio. 1001324

Johnson, M. D. (1994). Synaptic glutamate release by postnatal rat serotonergic neurons in microculture. Neuron 12, 433-442. doi: 10.1016/0896-6273(94) 90283-6

Junge, H. J., Rhee, J. S., Jahn, O., Varoqueaux, F., Spiess, J., Waxham, M. N., et al. (2004). Calmodulin and Munc13 form a $\mathrm{Ca}^{2+}$ sensor/effector complex that controls short-term synaptic plasticity. Cell 118, 389-401. doi: 10.1016/j.cell. 2004.06.029
Kaneda, M., Nakamura, H., and Akaike, N. (1988). Mechanical and enzymatic isolation of mammalian CNS neurons. Neurosci. Res. 5, 299-315. doi: 10.1016/0168-0102(88)90032-6

Karabelas, A. B., and Purpura, D. P. (1980). Evidence for autapses in the substantia nigra. Brain Res. 200, 467-473. doi: 10.1016/0006-8993(80)90935-x

Kay, A. R., and Wong, R. K. S. (1986). Isolation of neurons suitable for patchclamping from adult mammalian central nervous systems. J. Neurosci. Methods 16, 227-238. doi: 10.1016/0165-0270(86)90040-3

Kellogg, R., Mackie, K., and Straiker, A. (2009). Cannabinoid $\mathrm{CB}_{1}$ receptor-dependent long-term depression in autaptic excitatory neurons. J. Neurophysiol. 102, 1160-1171. doi: 10.1152/jn.00266.2009

Kimura, F., Otsu, Y., and Tsumoto, T. (1997). Presynaptically silent synapses: spontaneously active terminals without stimulus-evoked release demonstrated in cortical autapses. J. Neurophysiol. 77, 2805-2815. doi: 10.1152/jn.1997.77. 5.2805

Kumura, E., Kimura, F., Taniguchi, N., and Tsumoto, T. (2000). Brain-derived neurotrophic factor blocks long-term depression in solitary neurones cultured from rat visual cortex. J. Physiol. 524, 195-204. doi: 10.1111/j.1469-7793.2000. t01-2-00195.x

Landis, S. C. (1976). Rat sympathetic neurons and cardiac myocytes developing in microcultures: correlation of the fine structure of endings with neurotransmitter function in single neurons. Proc. Natl. Acad. Sci. U S A 73, 4220-4224. doi: 10.1073/pnas.73.11.4220

Lee, S.-H., Rosenmund, C., Schwaller, B., and Neher, E. (2000). Differences in $\mathrm{Ca}^{2+}$ buffering properties between excitatory and inhibitory hippocampal neurons from the rat. J. Physiol. 525, 405-418. doi: 10.1111/j.1469-7793.2000. t01-3-00405.x

Li, B., Chen, N., Luo, T., Otsu, Y., Murphy, T. H., and Raymond, L. A. (2002). Differential regulation of synaptic and extra-synaptic NMDA receptors. Nat. Neurosci. 5, 833-834. doi: 10.1038/nn912

Li, B., Otsu, Y., Murphy, T. H., and Raymond, L. A. (2003). Developmental decrease in NMDA receptor desensitization associated with shift to synapse and interaction with postsynaptic density-95. J. Neurosci. 23, 11244-11254. doi: 10.1523/JNEUROSCI.23-35-11244.2003

Li, Y., Schmid, G., Hänggi, P., and Schimansky-Geier, L. (2010). Spontaneous spiking in an autaptic Hodgkin-Huxley setup. Phys. Rev. E Stat. Nonlin. Soft Matter. Phys. 82:061907. doi: 10.1103/physreve.82.061907

Liu, H., Chapman, E. R., and Dean, C. (2013). “Self” versus “non-self” connectivity dictates properties of synaptic transmission and plasticity. PLoS One 8:e62414. doi: 10.1371/journal.pone.0062414

Liu, H., Dean, C., Arthur, C. P., Dong, M., and Chapman, E. R. (2009). Autapses and networks of hippocampal neurons exhibit distinct synaptic transmission phenotypes in the absence of synaptotagmin I. J. Neurosci. 29, 7395-7403. doi: 10.1523/JNEUROSCI.1341-09.2009

Lu, Z., Piechowicz, M., and Qiu, S. (2016). A simplified method for ultra-low density, long-term primary hippocampal neuron culture. J. Vis. Exp. 109:e53797. doi: 10.3791/53797

Lübke, J., Markram, H., Frotscher, M., and Sakmann, B. (1996). Frequency and dendritic distribution of autapses established by Layer 5 pyramidal neurons in the developing rat neocortex: comparison with synaptic innervation of adjacent neurons of the same class. J. Neurosci. 16, 3209-3218. doi: 10.1523/JNEUROSCI.16-10-03209.1996

Magistretti, J., de Curtis, M., Vescovi, A., Galli, R., and Gritti, A. (1996). Long-term survival of cortical neurones from adult guinea-pig maintained in low-density cultures. Neuroreport 7, 1559-1564. doi: 10.1097/00001756-199607080-00005

Manseau, F., Marinelli, S., Mendez, P., Schwaller, B., Prince, D. A., Huguenard, J. R., et al. (2010). Desynchronization of neocortical networks by asynchronous release of GABA at autaptic and synaptic contacts from fast-spiking interneurons. PLoS Biol. 8:e1000492. doi: 10.1371/journal.pbio. 1000492

Meijer, M., Cijsouw, T., Toonen, R. F., and Verhage, M. (2015). Synaptic effects of Munc18-1 alternative splicing in excitatory hippocampal neurons. PLoS One 10:e0138950. doi: 10.1371/journal.pone.0138950

Mennerick, S., Benz, A., and Zorumski, C. F. (1996). Components of glial responses to exogenous and synaptic glutamate in rat hippocampal microcultures. J. Neurosci. 16, 55-64. doi: 10.1523/JNEUROSCI.16-01-00 055.1996 
Mennerick, S., Que, J., Benz, A., and Zorumski, C. F. (1995). Passive and synaptic properties of hippocampal neurons grown in microcultures and in mass cultures. J. Neurophysiol. 73, 320-332. doi: 10.1152/jn.1995.73.1.320

Mennerick, S., Shen, W., Xu, W., Benz, A., Tanaka, K., Shimamoto, K., et al. (1999). Substrate turnover by transporters curtails synaptic glutamate transients. J. Neurosci. 19, 9242-9251. doi: 10.1523/JNEUROSCI.19-21-09242.1999

Mennerick, S., and Zorumski, C. F. (1994). Glial contributions to excitatory neurotransmission in cultured hippocampal cells. Nature 368, 59-62. doi: $10.1038 / 368059 \mathrm{a} 0$

Mennerick, S., and Zorumski, C. F. (1995a). Paired-pulse modulation of fast excitatory synaptic currents in microcultures of rat hippocampal neurons. J. Physiol. 488, 85-101. doi: 10.1113/jphysiol.1995.sp020948

Mennerick, S., and Zorumski, C. F. (1995b). Presynaptic influence on the time course of fast excitatory synaptic currents in cultured hippocampal cells. J. Neurosci. 15, 3178-3192. doi: 10.1523/JNEUROSCI.15-04-03178.1995

Michel, F. J., and Trudeau, L. E. (2000). Clozapine inhibits synaptic transmission at GABAergic synapses established by ventral tegmental area neurones in culture. Neuropharmacology 39, 1536-1543. doi: 10.1016/s0028-3908(99) 00239-7

Moechars, D., Weston, M. C., Leo, S., Callaerts-Vegh, Z., Goris, I., Daneels, G., et al. (2006). Vesicular glutamate transporter VGLUT2 expression levels control quantal size and neuropathic pain. J. Neurosci. 26, 12055-12066. doi: 10.1523/JNEUROSCI.2556-06.2006

Moulder, K. L., Jiang, X., Taylor, A. A., Shin, W., Gillis, K. D., and Mennerick, S. (2007). Vesicle pool heterogeneity at hippocampal glutamate and GABA synapses. J. Neurosci. 27, 9846-9854. doi: 10.1523/JNEUROSCI.280307.2007

Moulder, K. L., Meeks, J. P., Shute, A. A., Hamilton, C. K., de Erausquin, G., and Mennerick, S. (2004). Plastic elimination of functional glutamate release sites by depolarization. Neuron 42, 423-435. doi: 10.1016/s0896-6273(04) 00184-9

Moulder, K. L., and Mennerick, S. (2005). Reluctant vesicles contribute to the total readily releasable pool in glutamatergic hippocampal neurons. J. Neurosci. 25, 3842-3850. doi: 10.1523/JNEUROSCI.5231-04.2005

Murthy, V. N., Sejnowski, T. J., and Stevens, C. F. (1997). Heterogeneous release properties of visualized individual hippocampal synapses. Neuron 18, 599-612. doi: 10.1016/s0896-6273(00)80301-3

Murthy, V. N., Schikorski, T., Stevens, C. F., and Zhu, Y. (2001). Inactivity produces increases in neurotransmitter release and synapse size. Neuron 32, 673-682. doi: 10.1016/s0896-6273(01)00500-1

Murthy, V. N., and Stevens, C. F. (1998). Synaptic vesicles retain their identity through the endocytic cycle. Nature 392, 497-501. doi: 10.1038/ 33152

Nanou, E., Sullivan, J. M., Scheuer, T., and Catterall, W. A. (2016). Calcium sensor regulation of the $\mathrm{Ca}_{V} 2.1 \mathrm{Ca}^{2+}$ channel contributes to short-term synaptic plasticity in hippocampal neurons. Proc. Natl. Acad. Sci. U S A 113, 1062-1067. doi: 10.1073/pnas. 1524636113

Nishiki, T., and Augustine, G. J. (2004). Synaptotagmin I synchronizes transmitter release in mouse hippocampal neurons. J. Neurosci. 24, 6127-6132. doi: 10.1523/JNEUROSCI.1563-04.2004

Ogata, N., and Tatebayashi, H. (1991). Primary culture of neurons derived from the adult mammalian brain. Brain Res. Bull. 27, 747-750. doi: 10.1016/03619230(91)90058-r

Otsu, Y., and Murphy, T. H. (2004). Optical postsynaptic measurement of vesicle release rates for hippocampal synapses undergoing asynchronous release during train stimulation. J. Neurosci. 24, 9076-9086. doi: 10.1523/JNEUROSCI. 2060-04.2004

Otsu, Y., Shahrezaei, V., Li, B., Raymond, L. A., Delaney, K. R., and Murphy, T. H. (2004). Competition between phasic and asynchronous release for recovered synaptic vesicles at developing hippocampal autaptic synapses. J. Neurosci. 24, 420-433. doi: 10.1523/JNEUROSCI.4452-03.2004

Pan, Z. Z., Tong, G., and Jahr, C. E. (1993). A false transmitter at excitatory synapses. Neuron 11, 85-91. doi: 10.1016/0896-6273(93)90273-t

Paraskevopoulou, F., Herman, M. A., and Rosenmund, C. (2019). Glutamatergic innervation onto striatal neurons potentiates GABAergic synaptic output. J. Neurosci. 39, 4448-4460. doi: 10.1523/JNEUROSCI.263018.2019
Park, M. R., Lighthall, J. W., and Kitai, S. T. (1980). Recurrent inhibition in the rat neostriatum. Brain Res. 194, 359-369. doi: 10.1016/0006-8993(80) 91217-2

Pawelzik, H., Hughes, D. I., and Thomson, A. M. (2003). Modulation of inhibitory autapses and synapses on rat CA1 interneurones by $\mathrm{GABA}_{A}$ receptor ligands. J. Physiol. 546, 701-716. doi: 10.1113/jphysiol.2002.035121

Peters, A., and Proskauer, C. C. (1980). Synaptic relationships between a multipolar stellate cell and a pyramidal neuron in the rat visual cortex. A combined Golgi-electron microscope study. J. Neurocytology 9, 163-183. doi: 10.1007/bf01205156

Pouzat, C., and Marty, A. (1998). Autaptic inhibitory currents recorded from interneurones in rat cerebellar slices. J. Physiol. 509, 777-783. doi: 10.1111/j. 1469-7793.1998.777bm.x

Pouzat, C., and Marty, A. (1999). Somatic recording of GABAergic autoreceptor current in cerebellar stellate and basket cells. J. Neurosci. 19, 1675-1690. doi: 10.1523/JNEUROSCI.19-05-01675.1999

Prakriya, M., and Mennerick, S. (2000). Selective depression of low-release probability excitatory synapses by sodium channel blockers. Neuron 26 , 671-682. doi: 10.1016/s0896-6273(00)81203-9

Pratt, K. G., Zhu, P., Watari, H., Cook, D. G., and Sullivan, J. M. (2011). A novel role for $\gamma$-secretase: selective regulation of spontaneous neurotransmitter release from hippocampal neurons. J. Neurosci. 31, 899-906. doi: 10.1523/JNEUROSCI.4625-10.2011

Preston, R. J., Bishop, G. A., and Kitai, S. T. (1980). Medium spiny neuron projection from the rat striatum: an intracellular horseradish peroxidase study. Brain Res. 183, 253-263. doi: 10.1016/0006-8993(80)90462-x

Pyott, S. J., and Rosenmund, C. (2002). The effects of temperature on vesicular supply and release in autaptic cultures of rat and mouse hippocampal neurons. J. Physiol. 539, 523-535. doi: 10.1113/jphysiol.2001.013277

Raman, I. M., Tong, G., and Jahr, C. E. (1996). $\beta$-adrenergic regulation of synaptic NMDA receptors by cAMP-dependent protein kinase. Neuron 16, 415-421. doi: 10.1016/s0896-6273(00)80059-8

Rao, A., Cha, E. M., and Craig, A. M. (2000). Mismatched appositions of presynaptic and postsynaptic components in isolated hippocampal neurons. J. Neurosci. 20, 8344-8353. doi: 10.1523/JNEUROSCI.20-22-08344.2000

Reid, C. A., Bekkers, J. M., and Clements, J. D. (1998). N- and P/Q-type $\mathrm{Ca}^{+}$channels mediate transmitter release with a similar cooperativity at rat hippocampal autapses. J. Neurosci. 18, 2849-2855. doi: 10.1523/JNEUROSCI. 18-08-02849.1998

Reid, C. A., Clements, J. D., and Bekkers, J. M. (1997). Nonuniform distribution of $\mathrm{Ca}^{2+}$ channel subtypes on presynaptic terminals of excitatory synapses in hippocampal cultures. J. Neurosci. 17, 2738-2745. doi: 10.1523/JNEUROSCI. 17-08-02738.1997

Ricoult, S. G., Goldman, J. S., Stellwagen, D., Juncker, D., and Kennedy, T. E. (2012). Generation of microisland cultures using microcontact printing to pattern protein substrates. J. Neurosci. Methods 208, 10-17. doi: 10.1016/j. jneumeth.2012.04.016

Ripoli, C., Piacentini, R., Riccardi, E., Leone, L., Li Puma, D. D., Bitan, G., et al. (2013). Effects of different amyloid $\beta$-protein analogues on synaptic function. Neurobiol. Aging 34, 1032-1044. doi: 10.1016/j.neurobiolaging.2012. 06.027

Rosenmund, C., Clements, J. D., and Westbrook, G. L. (1993). Nonuniform probability of glutamate release at a hippocampal synapse. Science 262, 754-757. doi: 10.1126/science.7901909

Rosenmund, C., Sigler, A., Augustin, I., Reim, K., Brose, N., and Rhee, J.-S. (2002). Differential control of vesicle priming and short-term plasticity by Munc13 isoforms. Neuron 33, 411-424. doi: 10.1016/s0896-6273(02)00568-8

Rosenmund, C., and Stevens, C. F. (1996). Definition of the readily releasable pool of vesicles at hippocampal synapses. Neuron 16, 1197-1207. doi: 10.1016/s0896-6273(00)80146-4

Rost, B. R., Breustedt, J., Schoenherr, A., Grosse, G., Ahnert-Hilger, G., and Schmitz, D. (2010). Autaptic cultures of single hippocampal granule cells of mice and rats. Eur. J. Neurosci. 32, 939-947. doi: 10.1111/j.1460-9568.2010. 07387.x

Rost, B. R., Schneider, F., Grauel, M. K., Wozny, C., Bentz, C., Blessing, A., et al. (2015). Optogenetic acidification of synaptic vesicles and lysosomes. Nat. Neurosci. 18, 1845-1852. doi: 10.1038/nn.4161 
Schlüter, O. M., Schmitz, F., Jahn, R., Rosenmund, C., and Sudhof, T. C. (2004). A complete genetic analysis of neuronal Rab3 function. J. Neurosci. 24, 6629-6637. doi: 10.1523/JNEUROSCI.1610-04.2004

Schupp, M., Malsam, J., Ruiter, M., Scheutzow, A., Wierda, K. D., Sollner, T. H., et al. (2016). Interactions between SNAP-25 and synaptotagmin-1 are involved in vesicle priming, clamping spontaneous and stimulating evoked neurotransmission. J. Neurosci. 36, 11865-11880. doi: 10.1523/JNEUROSCI. 1011-16.2016

Segal, M. M. (1991). Epileptiform activity in microcultures containing one excitatory hippocampal neuron. J. Neurophysiol. 65, 761-770. doi: 10.1152/jn. 1991.65.4.761

Segal, M. M. (1994). Endogenous bursts underlie seizurelike activity in solitary excitatory hippocampal neurons in microcultures. J. Neurophysiol. 72, 1874-1884. doi: 10.1152/jn.1994.72.4.1874

Segal, M. M., Baughman, R. W., Jones, K. A., and Huettner, J. E. (1998). "Mass cultures and microislands of neurons from postnatal rat brain," in Culturing Nerve Cells, 2nd Edn. eds G. Banker and K. Goslin (Cambridge, MA: MIT Press), 309-338.

Segal, M. M., and Furshpan, E. J. (1990). Epileptiform activity in microcultures containing small numbers of hippocampal neurons. J. Neurophysiol. 64, 1390-1399. doi: 10.1152/jn.1990.64.5.1390

Sgro, A. E., Nowak, A. L., Austin, N. S., Custer, K. L., Allen, P. B., Chiu, D. T., et al. (2011). A high-throughput method for generating uniform microislands for autaptic neuronal cultures. J. Neurosci. Methods 198, 230-235. doi: 10.1016/j. jneumeth.2011.04.012

Shi, W.-X., and Rayport, S. (1994). GABA synapses formed in vitro by local axon collaterals of nucleus accumbens neurons. J. Neurosci. 14, 4548-4560. doi: 10.1523/JNEUROSCI.14-07-04548.1994

Sobieski, C., Jiang, X., Crawford, D. C., and Mennerick, S. (2015). Loss of local astrocyte support disrupts action potential propagation and glutamate release synchrony from unmyelinated hippocampal axon terminals in vitro. J. Neurosci. 35, 11105-11117. doi: 10.1523/JNEUROSCI.128915.2015

Stevens, C. F., and Sullivan, J. M. (1998). Regulation of the readily releasable vesicle pool by protein kinase C. Neuron 21, 885-893. doi: 10.1016/s08966273(00)80603-0

Stevens, C. F., and Sullivan, J. M. (2003). The synaptotagmin C2A domain is part of the calcium sensor controlling fast synaptic transmission. Neuron 39, 299-308. doi: 10.1016/s0896-6273(03)00432-x

Stevens, C. F., and Wesseling, J. F. (1998). Activity-dependent modulation of the rate at which synaptic vesicles become available to undergo exocytosis. Neuron 21, 415-424. doi: 10.1016/s0896-6273(00)80550-4

Stevens, C. F., and Wesseling, J. F. (1999a). Augmentation is a potentiation of the exocytotic process. Neuron 22, 139-146. doi: 10.1016/s0896-6273(00)80685-6

Stevens, C. F., and Wesseling, J. F. (1999b). Identification of a novel process limiting the rate of synaptic vesicle cycling at hippocampal synapses. Neuron 24, 1017-1028. doi: 10.1016/s0896-6273(00)81047-8

Stevens, C. F., and Williams, J. H. (2007). Discharge of the readily releasable pool with action potentials at hippocampal synapses. J. Neurophysiol. 98, 3221-3229. doi: 10.1152/jn.00857.2007

Straiker, A., and Mackie, K. (2005). Depolarization-induced suppression of excitation in murine autaptic hippocampal neurones. J. Physiol. 569, 501-517. doi: 10.1113/jphysiol.2005.091918

Straiker, A., and Mackie, K. (2007). Metabotropic suppression of excitation in murine autaptic hippocampal neurons. J. Physiol. 578, 773-785. doi: 10.1113/jphysiol.2006.117499

Straiker, A., and Mackie, K. (2009). Cannabinoid signaling in inhibitory autaptic hippocampal neurons. Neuroscience 163, 190-201. doi: 10.1016/j.neuroscience. 2009.06.004

Straiker, A., Dvorakova, M., Zimmowitch, A., and Mackie, K. (2018). Cannabidiol inhibits endocannabinoid signaling in autaptic hippocampal neurons. Mol. Pharmacol. 94, 743-748. doi: 10.1124/mol.118.111864

Sulzer, D., Joyce, M. P., Lin, L., Geldwert, D., Haber, S. N., Hattori, T., et al. (1998). Dopamine neurons make glutamatergic synapses in vitro. J. Neurosci. 18, 4588-4602. doi: 10.1523/JNEUROSCI.18-15-06057.1998

Tamás, G., Buhl, E. H., and Somogyi, P. (1997). Massive autaptic self-innervation of GABAergic neurons in cat visual cortex. J. Neurosci. 17, 6352-6364. doi: 10.1523/JNEUROSCI.17-16-06352.1997
Tarsa, L., and Goda, Y. (2002). Synaptophysin regulates activity-dependent synapse formation in cultured hippocampal neurons. Proc. Natl. Acad. Sci. U S A 99, 1012-1016. doi: 10.1073/pnas.022575999

Thakur, P., Stevens, D. R., Sheng, Z. H., and Rettig, J. (2004). Effects of PKA-mediated phosphorylation of Snapin on synaptic transmission in cultured hippocampal neurons. J. Neurosci. 24, 6476-6481. doi: 10.1523/JNEUROSCI. 0590-04.2004

Thomas, C. G., Miller, A. J., and Westbrook, G. L. (2006). Synaptic and extrasynaptic NMDA receptor NR2 subunits in cultured hippocampal neurons. J. Neurophysiol. 95, 1727-1734. doi: 10.1152/jn.00771.2005

Ting, J. T., Kelley, B. G., and Sullivan, J. M. (2006). Synaptotagmin IV does not alter excitatory fast synaptic transmission or fusion pore kinetics in mammalian CNS neurons. Adv. Exp. Med. Biol. 26, 372-380. doi: 10.1523/JNEUROSCI. 3997-05.2006

Tong, G., and Jahr, C. E. (1994a). Block of glutamate transporters potentiates postsynaptic excitation. Neuron 13, 1195-1203. doi: 10.1016/08966273(94)90057-4

Tong, G., and Jahr, C. E. (1994b). Multivesicular release from excitatory synapses of cultured hippocampal neurons. Neuron 12, 51-59. doi: 10.1016/08966273(94)90151-1

Tong, G., Malenka, R. C., and Nicoll, R. A. (1996). Long-term potentiation in cultures of single hippocampal granule cells: a presynaptic form of plasticity. Neuron 16, 1147-1157. doi: 10.1016/s0896-6273(00)80141-5

Tong, G., Shepherd, D., and Jahr, C. E. (1995). Synaptic desensitization of NMDA receptors by calcineurin. Science 267, 1510-1512. doi: 10.1126/science. 7878472

Tovar, K. R., and Westbrook, G. L. (1999). The incorporation of NMDA receptors with a distinct subunit composition at nascent hippocampal synapses in vitro. J. Neurosci. 19, 4180-4188. doi: 10.1523/JNEUROSCI.19-10-04 180.1999

Tovar, K. R., and Westbrook, G. L. (2002). Mobile NMDA receptors at hippocampal synapses. Neuron 34, 255-264. doi: 10.1016/s0896-6273(02) 00658-x

Ullian, E. M., Sapperstein, S. K., Christopherson, K. S., and Barres, B. A. (2001). Control of synapse number by glia. Science 291, 657-660. doi: 10.1126/science. 291.5504.657

Valente, P., Orlando, M., Raimondi, A., Benfenati, F., and Baldelli, P. (2016). Fine tuning of synaptic plasticity and filtering by GABA released from hippocampal autaptic granule cells. Cereb. Cortex 26, 1149-1167. doi: 10.1093/cercor/ bhu301

Van der Loos, H., and Glaser, E. M. (1972). Autapses in neocortex cerebri: synapses between a pyramidal cell's axon and its own dendrites. Brain Res. 48, 355-360. doi: 10.1016/0006-8993(72)90189-8

Varoqueaux, F., Sigler, A., Rhee, J. S., Brose, N., Enk, C., Reim, K., et al. (2002). Total arrest of spontaneous and evoked synaptic transmission but normal synaptogenesis in the absence of Munc13-mediated vesicle priming. Proc. Natl. Acad. Sci. U S A 99, 9037-9042. doi: 10.1073/pnas.122623799

Ventimiglia, R., and Lindsay, R. M. (1998). "Rat striatal neurons in low-density, serum-free culture," in Culturing Nerve Cells, 2nd Edn. eds G. Banker and K. Goslin (Cambridge, MA: MIT Press), 371-393.

Vyklicky, V., Smejkalova, T., Krausova, B., Balik, A., Korinek, M., Borovska, J., et al. (2016). Preferential inhibition of tonically over phasically activated NMDA receptors by pregnane derivatives. J. Neurosci. 36, 2161-2175. doi: 10.1523/JNEUROSCI.3181-15.2016

Weber, J. P., Toft-Bertelsen, T. L., Mohrmann, R., Delgado-Martinez, I., and Sørensen, J. B. (2014). Synaptotagmin-7 is an asynchronous calcium sensor for synaptic transmission in neurons expressing SNAP-23. PLoS One 9:e114033. doi: 10.1371/journal.pone.0114033

Weston, M. C., Chen, H., and Swann, J. W. (2014). Loss of mTOR repressors Tscl or Pten has divergent effects on excitatory and inhibitory synaptic transmission in single hippocampal neuron cultures. Front. Mol. Neurosci. 7:1. doi: 10.3389/fnmol.2014.00001

White, J. A., Chow, C. C., Ritt, J., Soto-Trevino, C., and Kopell, N. (1998). Synchronization and oscillatory dynamics in heterogeneous, mutually inhibited neurons. J. Comput. Neurosci. 5, 5-16. doi: 10.1023/a:10088413 25921

Wierda, K. D., and Sørensen, J. B. (2014). Innervation by a GABAergic neuron depresses spontaneous release in glutamatergic neurons and unveils 
the clamping phenotype of synaptotagmin-1. J. Neurosci. 34, 2100-2110. doi: 10.1523/JNEUROSCI.3934-13.2014

Wiles, L., Gu, S., Pasqualetti, F., Parvesse, B., Gabrieli, D., Bassett, D. S., et al. (2017). Autaptic connections shift network excitability and bursting. Sci. Rep. 7:44006. doi: 10.1038/srep44006

Williams, S. R. (2004). Spatial compartmentalization and functional impact of conductance in pyramidal neurons. Nat. Neurosci. 7, 961-967. doi: $10.1038 / \mathrm{nn} 1305$

Wojcik, S. M., Rhee, J. S., Herzog, E., Sigler, A., Jahn, R., Takamori, S., et al. (2004). An essential role for vesicular glutamate transporter 1 (VGLUT1) in postnatal development and control of quantal size. Proc. Natl. Acad. Sci. U S A 101, 7158-7163. doi: 10.1073/pnas.0401764101

Xia, P., Chen, H. S., Zhang, D., and Lipton, S. A. (2010). Memantine preferentially blocks extrasynaptic over synaptic NMDA receptor currents in hippocampal autapses. J. Neurosci. 30, 11246-11250. doi: 10.1523/JNEUROSCI.248810.2010

Yin, L., Zheng, R., Ke, W., He, Q., Zhang, Y., Li, J., et al. (2018). Autapses enhance bursting and coincidence detection in neocortical pyramidal cells. Nat. Commun. 9:4890. doi: 10.1038/s41467-018-07317-4

Young, S. M. Jr. (2005). Proteolysis of SNARE proteins alters facilitation and depression in a specific way. Proc. Natl. Acad. Sci. U S A 102, 2614-2619. doi: 10.1073/pnas.0409656102
Yuste, R. (2015). From the neuron doctrine to neural networks. Nat. Rev. Neurosci. 16, 487-497. doi: 10.1038/nrn3962

Zimmermann, J., Herman, M. A., and Rosenmund, C. (2015). Co-release of glutamate and GABA from single vesicles in GABAergic neurons exogenously expressing VGLUT3. Front. Synaptic Neurosci. 7:16. doi: 10.3389/fnsyn. 2015.00016

Zimmermann, J., Trimbuch, T., and Rosenmund, C. (2014). Synaptobrevin 1 mediates vesicle priming and evoked release in a subpopulation of hippocampal neurons. J. Neurophysiol. 112, 1559-1565. doi: 10.1152/jn. 00340.2014

Conflict of Interest: The author declares that the research was conducted in the absence of any commercial or financial relationships that could be construed as a potential conflict of interest.

Copyright (C) 2020 Bekkers. This is an open-access article distributed under the terms of the Creative Commons Attribution License (CC BY). The use, distribution or reproduction in other forums is permitted, provided the original author(s) and the copyright owner(s) are credited and that the original publication in this journal is cited, in accordance with accepted academic practice. No use, distribution or reproduction is permitted which does not comply with these terms. 